\title{
Comparative and Functional Analysis of the Widely Occurring Family of Nep1-Like Proteins
}

\author{
Stan Oome and Guido Van den Ackerveken \\ Plant-Microbe Interactions, Department of Biology, Utrecht University, Utrecht, The Netherlands and Centre for BioSystems \\ Genomics (CBSG), Wageningen, The Netherlands
}

Submitted 26 April 2014. Accepted 26 June 2014.

Nep1-like proteins (NLP) are best known for their cytotoxic activity in dicot plants. NLP are taxonomically widespread among microbes with very different lifestyles. To learn more about this enigmatic protein family, we analyzed more than 500 available NLP protein sequences from fungi, oomycetes, and bacteria. Phylogenetic clustering showed that, besides the previously documented two types, an additional, more divergent, third NLP type could be distinguished. By closely examining the three NLP types, we identified a noncytotoxic subgroup of type 1 NLP (designated type 1a), which have substitutions in amino acids making up a cation-binding pocket that is required for cytotoxicity. Type 2 NLP were found to contain a putative calcium-binding motif, which was shown to be required for cytotoxicity. Members of both type 1 and type 2 NLP were found to possess additional cysteine residues that, based on their predicted proximity, make up potential disulfide bridges that could provide additional stability to these secreted proteins. Type 1 and type 2 NLP, although both cytotoxic to plant cells, differ in their ability to induce necrosis when artificially targeted to different cellular compartments in planta, suggesting they have different mechanisms of cytotoxicity.

The 24-kDa necrosis-and-ethylene inducing peptide 1 (Nep1) of Fusarium oxysporum, which was isolated from culture filtrates of this plant-pathogenic fungus (Bailey 1995), is the founding member of a widely occurring protein family. The Nep1 protein induces necrosis and ethylene production when infiltrated into the extracellular space in leaves of dicot, but not monocot, plant species. Since the discovery of Nep1, many Nep1-like proteins (NLP) have been identified, mostly in plantrelated microorganisms of both prokaryotic (gram-negative and gram-positive bacteria) and eukaryotic (fungi and oomycetes) origin (Gijzen and Nürnberger 2006; Pemberton and Salmond 2004). As the NLP constituted a new family of proteins, a Pfam domain was defined based on NPP1, an NLP of Phytophthora parasitica (Pfam domain PF05630) (Fellbrich et al. 2002). A prominent feature of the NPP1 domain is a sevenamino-acid (heptapeptide) motif, GHRHDWE, which is most strongly conserved in NLP of different species. Based on ini-

Corresponding author: G. Van den Ackerveken;

E-mail: g.vandenackerveken@uu.nl

* The $\boldsymbol{e}$-Xtra logo stands for "electronic extra" and indicates that ten supplementary figures, two supplementary tables, and a supplementary data file are published online and that Figure 10 appears in color online.

(C) 2014 The American Phytopathological Society tial phylogenetic clustering, two types of NLP could be distinguished by the presence of two conserved cysteine residues in type 1 NLP and four in type 2 NLP (Gijzen and Nürnberger 2006). The vast majority of NLP have an N-terminal signal peptide (SP), suggesting they are secreted and function extracellularly. In planta expression of PsojNIP, a type 1 NLP of Phytophthora sojae, in soybean hypocotyls (Qutob et al. 2002) and Arabidopsis thaliana leaves (Qutob et al. 2006) showed that the protein only caused necrosis, or cell death, when containing its SP but not when expressed without it. This suggested that the NLP acts extracellular of the plant cell to confer its cytotoxic activity.

The mechanism by which NLP induce necrosis is poorly understood. The main question is whether NLP-induced necrosis is caused by stimulation of the plant's immune system and associated programmed cell death or by direct toxicity of the NLP through disruption of the plant membrane (Gijzen and Nürnberger 2006). Application of recombinant NLP to $A$. thaliana leads to rapid activation of genes associated with defense and cell death (Bae et al. 2006; Qutob et al. 2006), suggesting an active role of the plant in necrosis induction. This was emphasized by the finding that necrosis induction by NPP1 in tobacco requires light and an active host metabolism (Qutob et al. 2006). If NLP would induce necrosis because of their recognition by a receptor, it would likely require only a small conserved peptide fragment (Boller and Felix 2009). However, induction of necrosis by NLP requires the proteins to be mostly intact (Veit et al. 2001), as minimal truncations on either of the N- or C- termini of, for instance, NPP1 of Phytophthora parasitica prevent it from inducing necrosis in plants (Fellbrich et al. 2002). Ottmann and associates (2009) showed that NLP permeabilize dicot-derived membrane vesicles in vitro, suggesting that cytotoxicity is the result of membrane leakage, which in turn activates defense responses and cell death. Monocot-derived membrane vesicles were not permeabilized, suggesting NLP cytotoxicity requires a dicot-specific target protein or membrane architecture. The cytolytic activity suggested the protein could form a pore in the plant membrane. However, evidence for a pore-forming activity is lacking so that the exact molecular mechanism of NLP cytotoxicity remains unknown.

The protein structures of two type 1 NLP have been published to date: $\mathrm{NLP}_{\mathrm{Pya}}$ of the oomycete Pythium aphanidermatum (PDB:3GNU) (Ottmann et al. 2009) and MpNEP2 of the Basidiomycete fungus Moniliophthora perniciosa (PDB:3ST1) (Zaparoli et al. 2011). These structures revealed that NLP adopt a fold similar to that of pore-forming Actinoporins (Ottmann et al. 2009). The two cysteines that are conserved and essential for induction of necrosis in type 1 NLP (Fellbrich et al. 2002) were confirmed to form a disulfide bridge. Further- 
more, three residues (underlined) of the highly conserved GHRHDWE heptapeptide motif were found to be part of an acidic cation-binding pocket. Substitution of these conserved histidine $(\mathrm{H})$, aspartic acid (D), and glutamic acid (E) residues in $\mathrm{NLP}_{\text {Pya }}$ by alanine disabled the induction of necrosis and membrane permeabilization by the protein, suggesting cationbinding is essential for these activities (Ottmann et al. 2009). The acidic pocket was proposed to interact with polar head groups of membrane lipids, thereby damaging or interacting with the plant membrane (Küfner et al. 2009).

NLP could contribute to the virulence of pathogenic microorganisms, e.g., $\mathrm{NLP}_{\mathrm{Pcc}}$ of the soft-rot bacterium Erwinia (Pectobacterium) carotovora subsp. carotovora, which was shown to be essential for virulence on potato tubers (Mattinen et al. 2004). In contrast, virulence of the wheat pathogen $\mathrm{Myco}$ sphaerella graminicola was not affected by deletion of its sole NLP gene (Motteram et al. 2009). Also, in several (fungal) species containing multiple NLP, the loss of a single NLP gene did not affect virulence (Arenas et al. 2010; Santhanam et al. 2013; Staats et al. 2007; Zhou et al. 2012), suggesting NLP do not play an essential role in virulence of these organisms. Alternatively, the loss of a single NLP gene in these species may not result in reduced virulence due to genetic redundancy.

In addition to cytotoxic NLP, many noncytotoxic NLP have been described that do not induce necrosis. Two of three tested NLP of Phytophthora infestans (Kanneganti et al. 2006), 11 of the 19 tested NLP of Phytophthora sojae (Dong et al. 2012), all 12 NLP of Hyaloperonospora arabidopsidis (Cabral et al. 2012), five of the seven full-length NLP of Verticillium dahliae (Zhou et al. 2012), and one of two tested NLP of Colletotrichum higginsianum (Kleemann et al. 2012) did not cause necrosis when transiently expressed in plants. The genes encoding noncytotoxic NLP were found to be expressed during the biotrophic and early stages of infection by these (hemi) biotrophic pathogens (Cabral et al. 2012; Dong et al. 2012; Kanneganti et al. 2006; Kleemann et al. 2012), suggesting they play a role during penetration or establishment of infection.

As many new microbial genome sequences became available in the last years, we bioinformatically analyzed the family of NLP by combining phylogenetic analyses and available structural information. Our results suggest that the NLP family consists of three distinct types, each with specific characteristics. We identified an important putative calcium-binding site in type 2 NLP that clearly distinguishes them from type 1 and type 3 NLP. Our study highlights the diversity in phylogenetic distribution, protein sequence, and function of NLP.

\section{RESULTS AND DISCUSSION}

\section{NLP group in three distinct subfamilies.}

Two proteins that represent both described types of NLP, the type $1 \mathrm{NLP}_{\mathrm{Pya}}$ of the oomycete Pythium aphanidermatum and the type $2 \mathrm{NLP}_{\mathrm{Pcc}}$ of the bacterium Pectobacterium carotovorum, formed the starting point of our search for NLP homologs. Exhaustive screening of DNA and protein databases for NLP family members using Blast (detailed below) resulted in a collection of 533 NLP homologs from more than 150 different species of bacteria, fungi, and oomycetes (Supplementary Data; Supplementary Table S1), yet not from any other taxonomic group. Phylogenetic analysis of all collected NLP resulted in a neighbor-joining tree (Fig. 1A) that showed a clearly distinguishable third group of NLP proteins (designated type
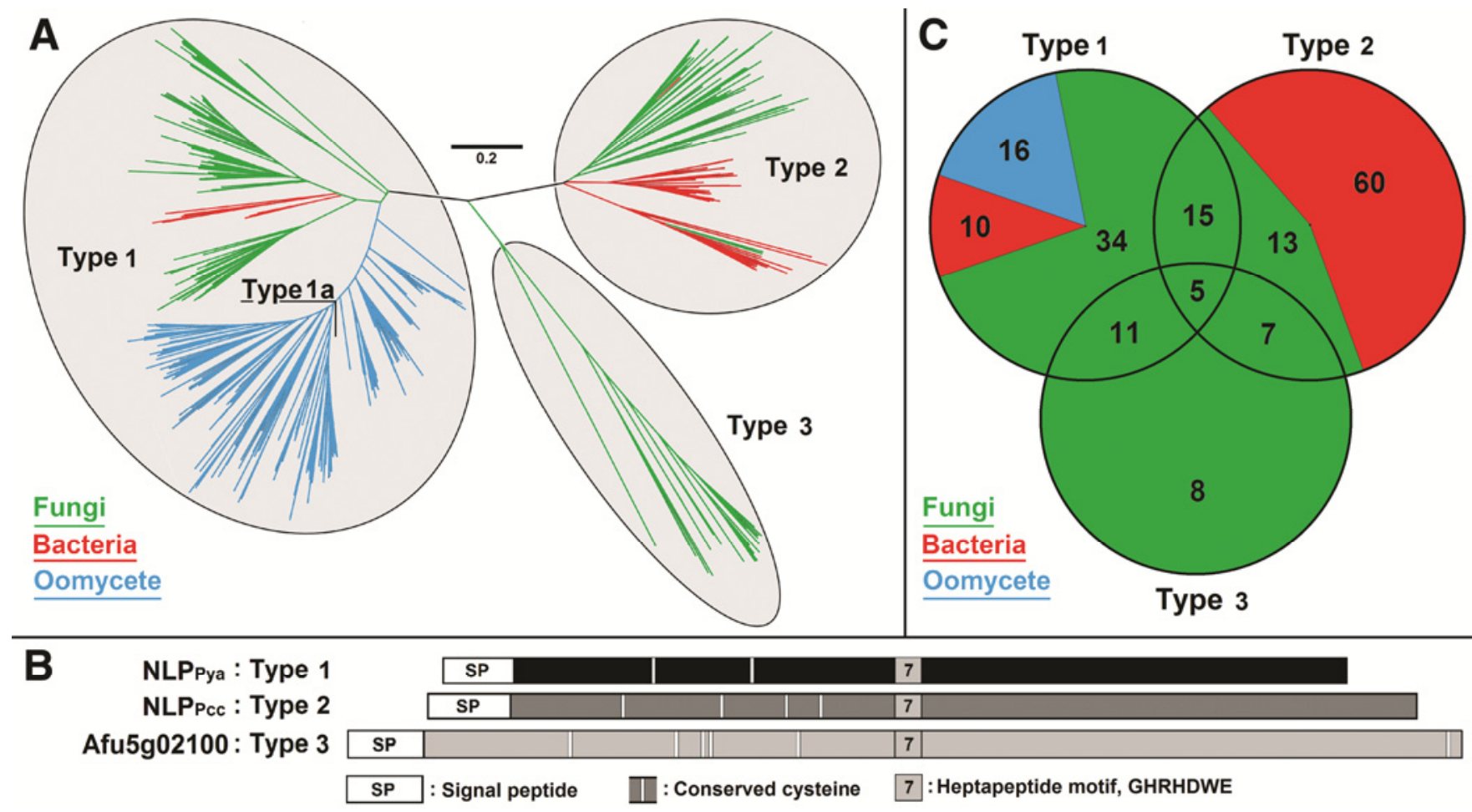

Fig. 1. Phylogenetic comparison and species distribution of Nep1-like proteins (NLP). A, Unrooted neighbor-joining protein-based tree of 231 oomycete (99 type 1, 132 type 1a), 135 fungal, and 12 bacterial type 1 NLP, 61 fungal and 61 bacterial type 2 NLP, and 33 fungal type 3 NLP. All amino acid sequences (without a signal peptide [SP]) were aligned using MEGA5; the tree was constructed with FigTree. B, Schematic representation of the three representatives of the three types of NLP: type 1 NLP are represented by NLP $_{\mathrm{Pya}}$ from Pythium aphanidermatum, type 2 NLP by NLP $\mathrm{P}_{\mathrm{Pcc}}$ from Pectobacterium carotovorum, and type 3 by Afu5g02100 from Aspergillus fumigatus. The NLP are characterized by the presence of a SP, conserved cysteines, and a seven-amino acid "GHRHDWE" motif or variant thereof. C, Numbers of species and the distribution of NLP types among species. Type 1 NLP are found in 91 species, type 2 NLP are found in 100 species, and type 3 NLP are found in 31 species. Fungi can have several combinations of the three types of NLP; although both type 1 and type 2 NLP are found in bacteria, no species of bacteria were found harboring more than one type of NLP. 
3 ) in addition to the previously described types 1 and 2 NLP (Gijzen and Nürnberger 2006). The type 3 NLP are more divergent from types 1 and 2, sharing only a central stretch of 50 amino acids with them, including the highly conserved heptapeptide motif. Sequences N- and C-terminal of these 50 amino acids in the type 3 NLP are very distinct from those of type 1 and type 2 NLP and are, therefore, not comparable. When performing the phylogenetic analysis using the conserved 50 amino-acid central domain of all NLP proteins, the three NLP types still clustered in the same three branches, indicating the type 3 NLP are not a subgroup of the other NLP types (Supplementary Fig. S1).

Of all our 533 collected NLP, 95\% carry a SP for secretion, all of them the heptapeptide motif (or variant thereof), and more than $98 \%$ of the type 1 and type 2 NLP contain the two conserved cysteine residues that form the disulfide bond in the published NLP structures. Most type 2 NLP have two additional cysteine residues (total of four), while only the type 2 NLP identified in members of genus Bacillus lack these. Type 3 NLP are characterized by a total of six cysteine residues, but these are in the $\mathrm{N}$ - and $\mathrm{C}$-terminal part of the proteins that share no similarity to type 1 and type 2 NLP and can, thus, not be aligned with the conserved cysteines in type 1 and type 2 NLP. All type 1 and type 2 NLP match the NPP1 family Pfam domain (PF05630), whereas this is only the case with $66 \%$ of the type 3 NLP. The overall architecture of the three NLP types is shown for a representative member of each type, the type $1 \mathrm{NLP}_{\text {Pya }}$, the type $2 \mathrm{NLP}_{\mathrm{Pcc}}$, and the type $3 \mathrm{NLP}$ of the fungus Aspergillus fumigatus (Afu5g02100) (Fig. 1B), including the location of the conserved cysteines and heptapeptide motif for all three NLP types.

The distribution of NLP across taxa differs between the three types (Fig. 1C). Whereas type 1 NLP are found in bacteria, fungi, and oomycetes, type 2 NLP are found in bacteria and fungi but not in oomycetes. Type 3 NLP have the narrowest distribution and have only been identified in a restricted number of fungal species and do not occur in bacteria or oomycetes. Sixty bacterial species contain a type 2 NLP, only ten contain a type 1 NLP, and, contrary to fungi, none contain both a type 1 and type 2 NLP (Fig. 1C). It is also important to note that the number of bacterial species having one or more NLP is rather limited, knowing that several thousands of bacterial genomes have been sequenced. In oomycetes, only type 1 NLP are found, which form a large clade as a result of expansion of the number of $N L P$ genes within oomycete species belonging to the plant-infecting order Peronosporales.

In fungi, species with all possible combinations of the three types of NLP are found. The genomes of five fungal species encode all three NLP types. Phylogenetic clustering of these fungal NLP resulted in three major clades (Supplementary Fig. S2) fitting the three NLP types shown in Figure 1A. The fact that the three types only occur together in fungal species belonging to the Ascomycetes suggests that the evolutionary origin of the NLP is in this phylum. Duplication of an ancestral NLP gene and diversification events then led to the three types that expanded even further in a selection of fungal species, mostly plant-associated ones. Oomycete NLP all cluster together, suggesting a single horizontal gene transfer (HGT) event of an Ascomycete fungus type $1 N L P$ to a common ancestor of members of the order Peronosporales, which then further expanded within this lineage. The latter hypothesis was also proposed by Richards and associates (2011), as NLP are one of the 20 gene families predicted to be acquired by oomycetes from fungi through HGT. HGT is also the most plausible explanation for the occurrence of type 2 NLP in a limited number of bacterial species belonging to the Gammaproteobacteria and Actinobacteria.
Type 3 NLP are specific for ascomycetes.

Type 3 NLP are only found in orders of the Ascomycete fungi (Supplementary Fig. S3). With 33 members identified thus far, they are not as widespread as types 1 and 2 NLP. The fungi containing type 3 NLP have many different lifestyles; in addition to plant pathogens, type 3 NLP are, among others, found in the plant endosymbiont Oidiodendron maius, the nematode pathogen Arthrobotrys oligospora, the bat pathogen Geomyces destructans, and the coprophilous Ascobolus immersus. In the latter species, which lacks type 1 and type 2 NLP, we find the highest number (three) of type 3 NLP of all examined species. Little is known about these proteins, and thus far, experimental data is only available for the type 3 NLP (Afu5g02100) of the opportunistic human pathogen Aspergillus fumigatus, which was identified in a proteomics analysis of secreted fibrinogen-binding proteins (Upadhyay et al. 2012). Preliminary data suggest that expression of the Verticillium dahliae type 3 NLP VDAG_07972T0 is induced during infection of tomato (P. Santhanam and B. Thomma personal communication). Also, expression of a type $3 N L P$ was observed during infection of insects by Metarhizium anisopliae (EFY97649) and M. acridum (EFY91472) (Gao et al. 2011; C. Wang personal communication).

\section{Type 2 NLP are widely distributed \\ in fungi and bacteria with various lifestyles.}

Fungal type 2 NLP are found in many orders of the Ascomycota (Fig. 2), and in one species of the primitive chytrid fungi (Gonapodya prolifera, phylum Monoblepharidomycetes). Based on its phylogenetic clustering, the latter likely obtained a type 2 NLP from a Gammaproteobacterium (Fig. 2). Type 2 NLP are widely spread in two groups of bacteria; they were identified in species from several orders of Gammaproteobacteria and in species of the phylogenetically distantly related order of Actinomycetales. The only exception to this is a type 2 NLP homolog identified in a small number of species from the genus Bacillus (phylum Firmicutes). These are also the only type 2 NLP lacking the characteristic second disulfide bridge. In addition, these Bacillus NLP are fused to a lectin domain that shares homology with ricin B (Schouten et al. 2008). Their clustering with the NLP of the distantly related Actinobacteria and their G/C content (Supplementary Fig. S4) suggest HGT from a Streptomyces species to these Bacillus species. Another possible HGT is likely responsible for the occurrence of a type 2 NLP in a Streptomyces species (Streptomyces sp. strain Mg1_2) that clusters within the Ascomycete NLP.

Type 2 NLP are found in plant-associated microbes, e.g., the necrotrophic bacterium Pectobacterium carotovorum, the dicot plant pathogen Nectria haematococca, and the monocot symbiont Epichloë festucae, but also in non-plant associated microbes, e.g., in the coprophilous Podospora anserina. Interestingly, NLP were also found in insect pathogenic fungi of the genera Beauveria, Cordiceps, and Metarhizium, which have also been isolated as plant endophytes (Vega et al. 2009). During infection of insects by the fungi $M$. anisopliae and $M$. acridum, expression of type 2 NLP was detected at 24 h postinoculation (Gao et al. 2011; C. Wang personal communication), suggesting these proteins may play a role in insect pathogenesis. Animal cell cytolysis caused by NLP has been tested on sheep erythrocytes. However, no hemolysis was observed in response to the type 1 NLP PpNPP1 of Phytophthora parasit$i c a$, which is cytolytic in dicot plants (Qutob et al. 2006). Also, other animal-related microorganisms harbor type 2 NLP, e.g., the coral pathogen Vibrio coralliilyticus (O de Santos et al. 2011) and the bivalve endosymbiont Teredinibacter turnerae (Yang et al. 2009). Strikingly, type 2 NLP are also found in the 


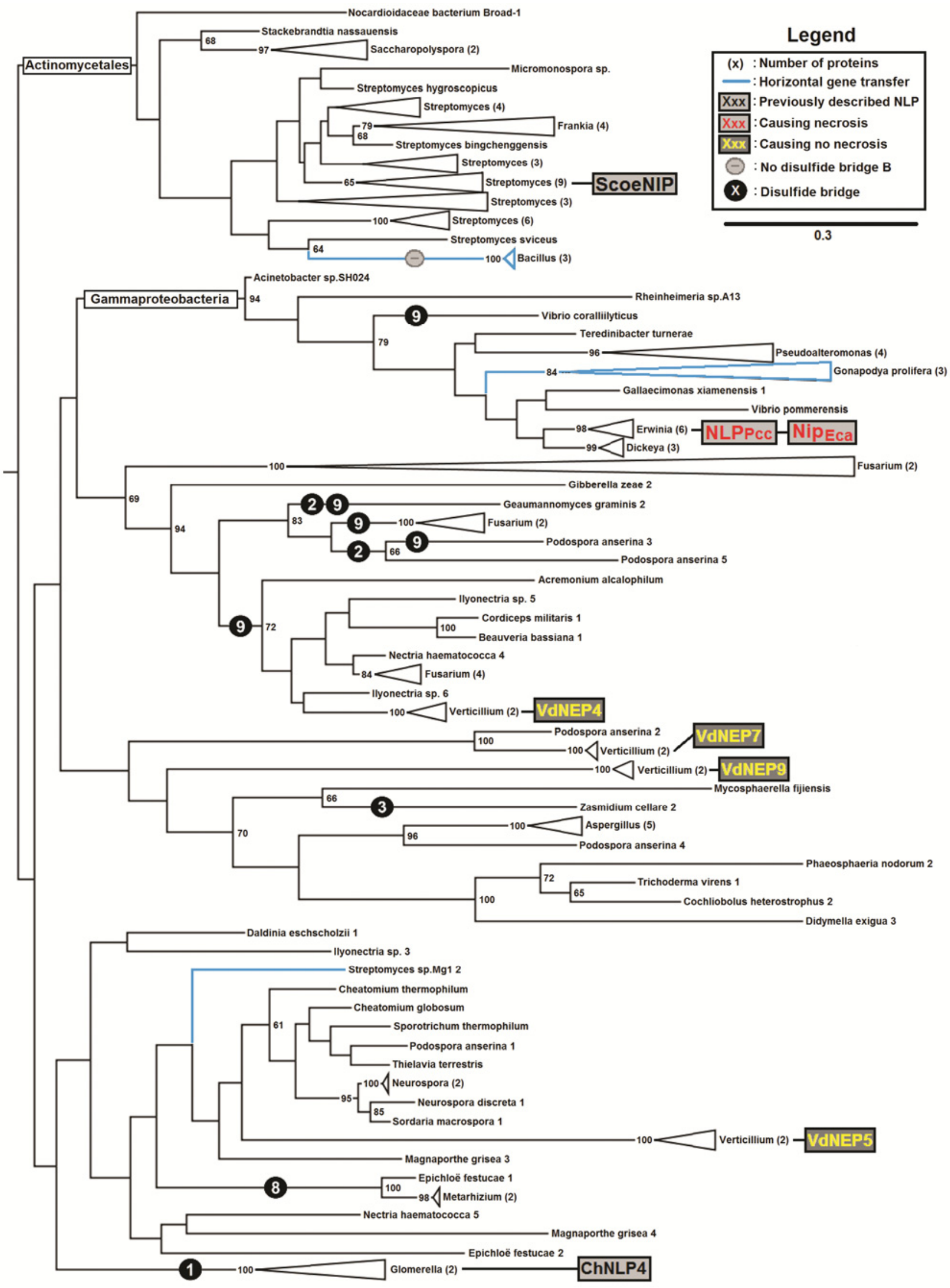

Fig. 2. Phylogenetic distribution of type 2 Nep1-like proteins (NLP). Aspergillus includes Neosartoria spp., Glomerella includes Colletotrichum spp.,

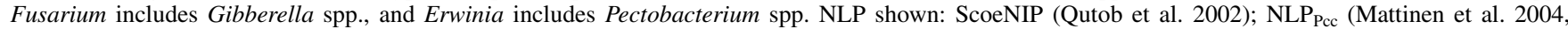
Ottmann et al. 2009); Nip Eca (Pemberton et al. 2005); ChNLP4 (Kleemann et al. 2012); VdNEP4, VdNEP5, VdNEP7, VdNEP9 (Zhou et al. 2012). Additional disulfide bridges found in the indicated branches are discussed below. Unrooted maximum likelihood tree of 61 fungal and 61 bacterial type 2 NLP. Only bootstrap values $>60$ are shown. 
animal-associated Bacillus cereus and B. thuringiensis (Jensen et al. 2003), while type 1 NLP are only found in this genus in the plant-associated $B$. subtilis (Earl et al. 2008), its close relative $B$. licheniformis, and the alkalophylic plant litter-degrading B. halodurans (Takami et al. 2000). Only two of the six tested type 2 NLP have been shown to induce necrosis, NLP $_{\text {Ecc }}$
$\left(\mathrm{NLP}_{\mathrm{Pcc}}\right)$ from Pectobacterium (Erwinia) carotovorum subsp. carotovorum (Mattinen et al. 2004) and $\mathrm{NLP}_{\mathrm{Eca}}$ from Pectobacterium carotovorum subsp. atrosepticum (Pemberton et al. 2005). None of the four type 2 NLP of Verticillium dahliae induce necrosis when transiently expressed in plants (Zhou et al. 2012).

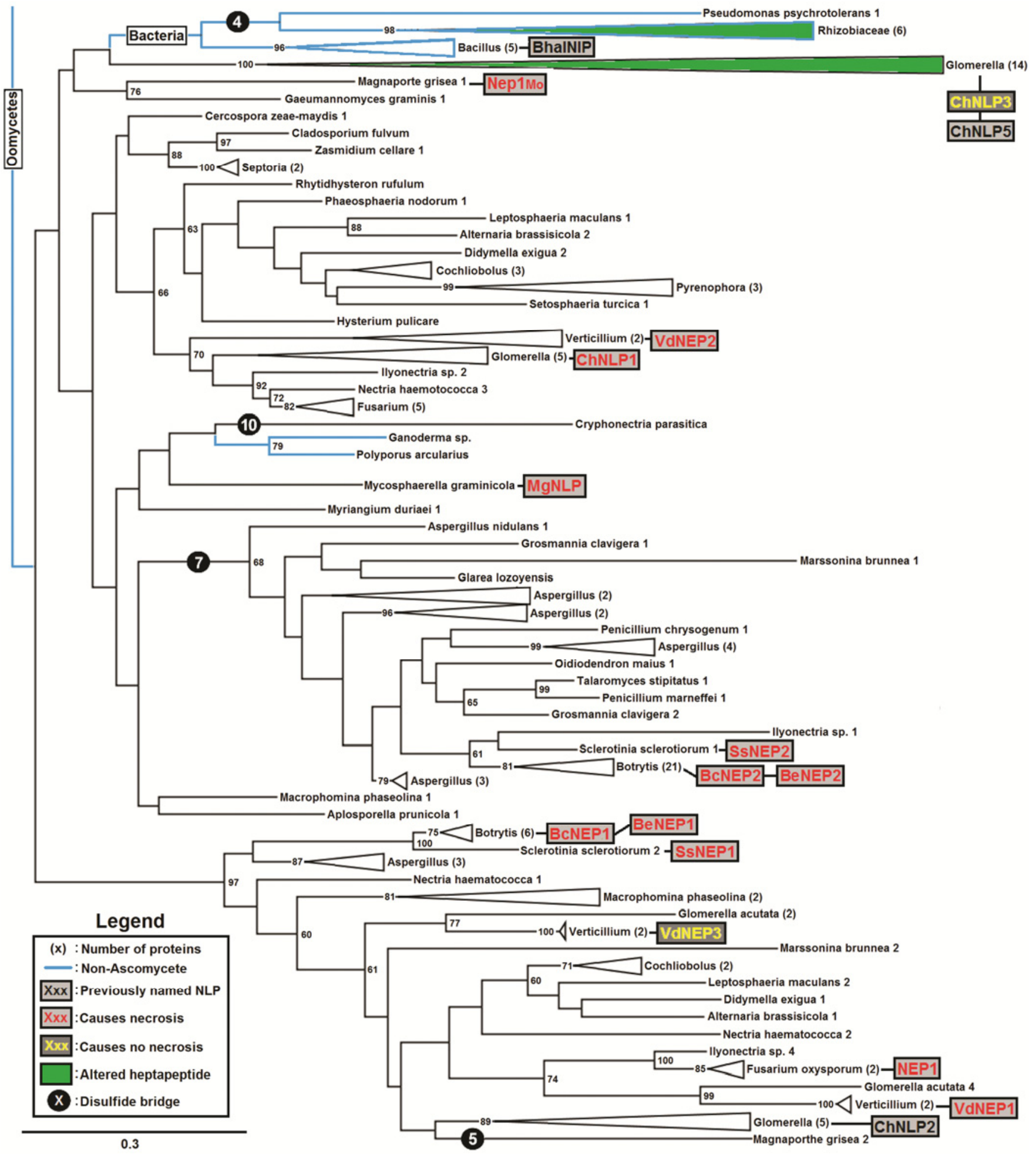

Fig. 3. Phylogenetic relationship between fungal and bacterial type 1 Nep1-like proteins (NLP). Aspergillus includes Neosartoria spp., Glomerella includes Colletotrichum spp., Botrytis includes Botryotinia spp., and Fusarium includes Gibberella spp. NLP shown: NEP1 (Bailey 1995); BhalNIP (Qutob et al. 2002); BeNEP1, BeNEP2 (Staats et al. 2007); BcNEP1, BcNEP2 (Schouten et al. 2008); MgNLP (Motteram et al. 2009); SsNEP1, SsNEP2 (Bashi et al 2010); VdNEP1, VdNEP2, VdNEP3 (Zhou et al. 2012); ChNLP1, ChNLP2, ChNLP3, ChNLP5 (Kleemann et al. 2012); Nep1 Mo (Zhang et al .2012). Additional disulfide bridges found in the indicated branches are discussed below. Unrooted maximum likelihood tree of 135 fungal and 12 bacterial type 1 NLP. Only bootstrap values $>60$ are shown. 


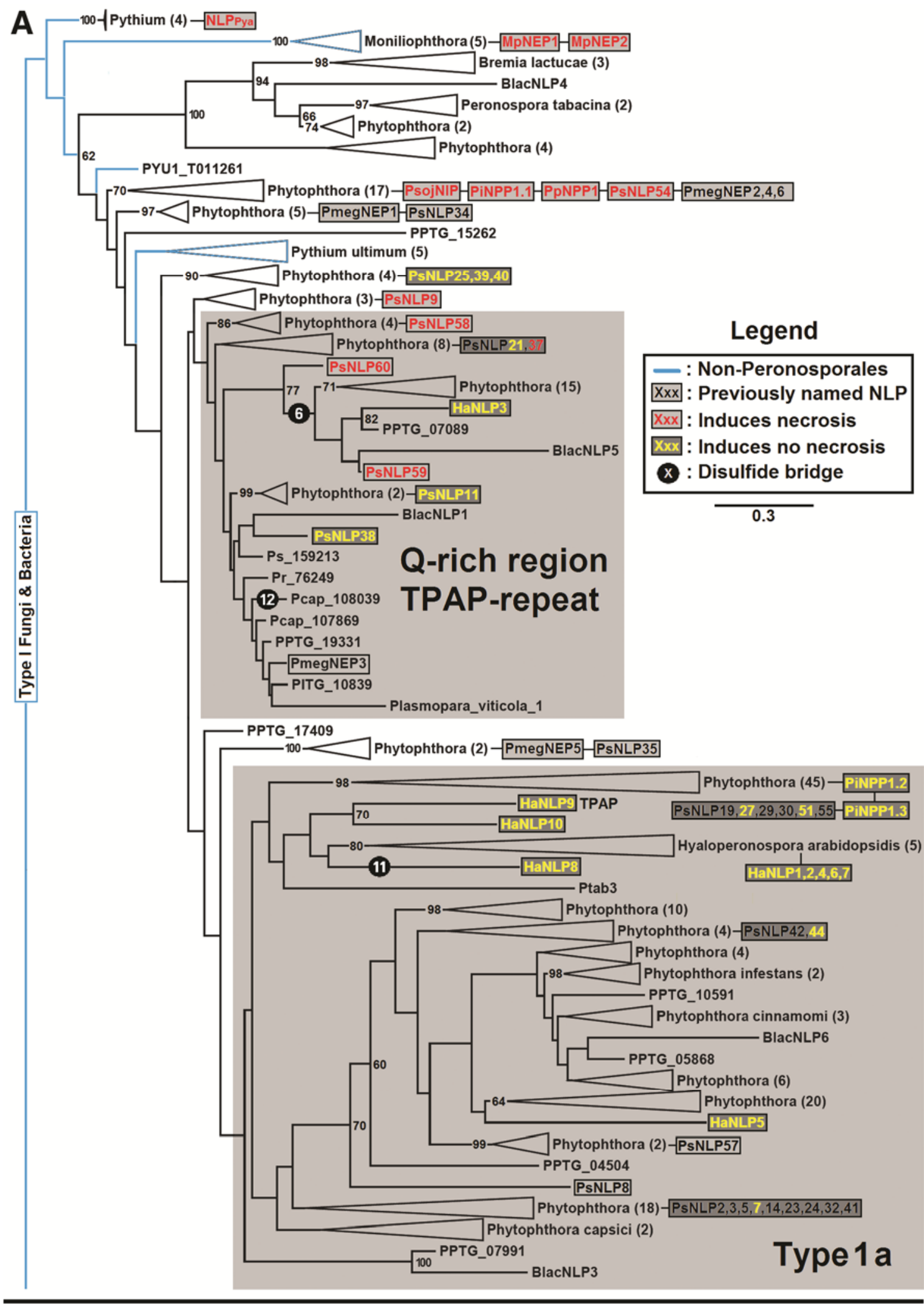

B

\begin{tabular}{l|l|l|l|l}
\hline SP & Q-rich & TPAP \\
\hline
\end{tabular}

Fig. 4. Phylogenetic distribution of Nep1-like proteins (NLP) in oomycetes. A, Unrooted maximum likelihood tree of 231 type 1 oomycete NLP. The tree can be divided in three groups: type 1 NLP, type 1 NLP with an added region consisting of one or both a Q-rich regionand a TPAP repeat, and type 1a NLP. 'Phytophthora' contains P. infestans, P. capsici, P. sojae, P. ramorum, P. parasitica, P. megakarya, and P. cinnamomi. NLP shown: PmegNEP1-6 (Bae et al. 2005); PiNPP1.1, PiNPP1.2, PiNPP1.3 (Kanneganti et al. 2006); MpNEP1, MpNEP2 (Garcia et al. 2007); PpNPP1, NLP Pya $(O t t m a n n$ et al. 2009); PsojNIP, HaNLP1-10 (Cabral et al. 2012); PsNLP1, 2, 3, 5,7 , , ,9, 11, 14, 19, 21, 23, 24, 25, 27, 29, 30, 32, 35, 37, 38, 39, 40, 41, 42, 44, 51, 54, 55, 57, 58, 59, and 60 (Dong et al. 2012). Additional disulfide bridges found in the indicated branches are discussed below. Only bootstrap values $>60$ are shown. B, Schematic representation of an NLP with a Q-rich region and TPAP-repeat, indicating the relative positions of the Q-rich region and TPAP repeat. 
Type 1 NLP are found mostly in plant-associated species.

Type 1 NLP form a monophyletic group and can be divided into fungal/bacterial NLP and oomycete NLP (Fig. 1A). A closer look at the fungal/bacterial NLP (Fig. 3) shows most of the fungal type 1 NLP are identified in many orders of the phylum Ascomycota. In this phylum, type 1 NLP are found mostly in genera with plant-associated lifestyles, ranging from the symbiotic (endomycorrhizal) genus Oidiodendron to the necrotrophic Sclerotinia and Botrytis pathogens. The only included fungal species not directly related to plants is the saprobic wine cellar fungus Zasmidium cellare (yet it belongs to the family Mycosphaerellaceae, which contains many plant pathogens [Crous et al. 2009]). Surprisingly, as monocots are insensitive to NLP (Bailey 1995), NLP genes have been found in fungi that solely interact with monocot plants, e.g., the grass pathogens belonging to the genera Pyrenophora, Cochliobolus, Setosphaeria, and Magnaporthe. This suggests that, in these species, cytotoxic type 1 NLP may have a function other than killing host cells. Only two fungal NLP in this group are found in species of phylum Basidiomycota, i.e., Polyporus arcularius and Ganoderma sp., which are both wood-degrading species of the order of Polyporales.
Bacterial species containing type 1 NLP are not very numerous. Phylogenetic distribution suggests that, in the evolutionary past, a type $1 N L P$ gene was acquired by a bacterial species, from which it was subsequently transferred to other bacterial species. Type 1 NLP are found in a few distantly related species of Proteobacteria (Rhizobiaceae and a single Pseudomonas species) and Firmicutes (Bacillus species). Of these bacteria, only Pseudomonas psychrotolerans (Hauser et al. 2004) may not be directly plant-associated.

Of 14 tested fungal type 1 NLP described in literature, 12 were found to induce necrosis. The heptapeptide is highly conserved in most bacterial and fungal NLP, except for the NLP found in the Rhizobiaceae family and a branch of type 1 NLP found in the genus Glomerella/Colletotrichum. The only tested NLP of this last group (ChNLP3 from Colletotrichum higginsianum) did not induce necrosis, as tested by transient expression in Nicotiana benthamiana leaves (Kleemann et al. 2012).

\section{Diversification of oomycete type 1 NLP.}

In oomycetes, NLP are only found in the Pythiaceae and Peronosporaceae families (Fig. 4A) of the Peronosporales order. They are, so far, not found in the third family of this order, the

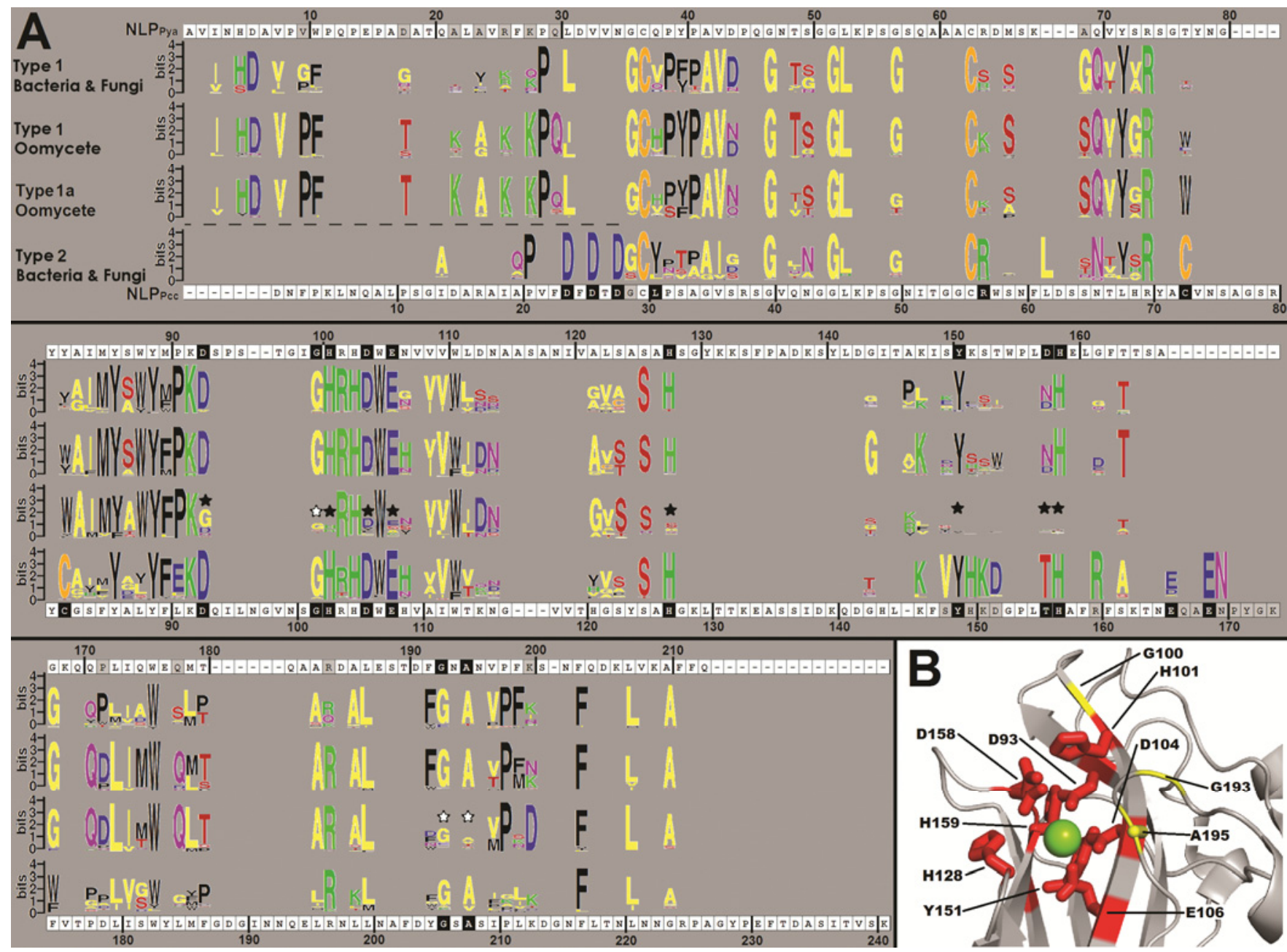

Fig. 5. Comparison of three groups of type 1 Nep1-like proteins (NLP) and all type 2 NLP sequences. A, Aligned Weblogos of the consensus sequence of 99 oomycete type 1 NLP, 132 oomycete type 1a NLP, 135 fungal and bacterial type 1 NLP and 122 type 2 NLP. The type 1 consensus Weblogos are aligned to $\mathrm{NLP}_{\text {Pya }}$ (3GNU), and the type 2 NLP consensus Weblogo is aligned to NLP $P_{\mathrm{Pcc}}$. Specific residues discussed in the text are marked in black or gray in the NLP $P_{\text {Pya }}$

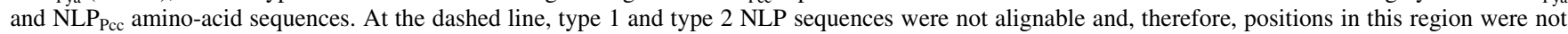
compared. The black stars indicate the eight residues that make up an acidic cation-binding pocket. White stars indicate three residues close this pocket, which are conserved in type 1 and type 2 NLP but not in type 1a. B, Three-dimensional model of the eight residues (red) making up the charged pocket in $\mathrm{NLP}_{\text {Pya }}$ (3GNZ) coordinating a magnesium ion (green). Yellow residues indicate the three positions conserved in other type 1 and type 2 NLP but not in type 1a NLP. Image was created using Polyview3D. 
Albuginaceae, as the genomes of both Albugo candida (Links et al. 2011) and Albugo laibachii (Kemen et al. 2011) completely lack NLP genes. All oomycete NLP form a separate branch with a common origin (Figs. 1A and 4A), and are not found together with fungal or bacterial NLP, suggesting that oomycetes have acquired these genes only once. The only exceptions are the NLP of the Basidiomycete fungi Moniliophthora perniciosa and Moniliophthora roreri, which group together with the oomycete proteins (Fig. 4A), suggesting they are the result of HGT. Based on an aberrant $\mathrm{G} / \mathrm{C}$ content, this gene transfer has likely occurred from an oomycete to these fungi (Tiburcio et al. 2010). The oomycete NLP are thus far exclusively found in plantpathogens, e.g., the necrotrophic Pythium, the hemibiotrophic Phytophthora, and the obligate biotrophic Peronospora, Hyaloperonospora, Bremia, and Plasmopara genera. Many oomycete NLP do not induce necrosis (Fig. 4A); of 32 tested NLP, only 10 are cytotoxic, Phytophthora infestans (Kanneganti et al. 2006), Phytophthora sojae (Dong et al. 2012), and H. arabidopsidis (Cabral et al. 2012) all express NLP during infections that have been shown not to induce necrosis.

In the Peronosporaceae, diversification of type 1 NLP can be observed (Fig. 4A). A subgroup of proteins has an additional hydrophilic domain between the SP and NPP1 domain. Within this "glutamine and proline-rich hydrophilic domain" (Gijzen and Nürnberger 2006), two regions are distinguished: i) a glutamine (Q)-rich region and ii) a TPAP repeat (Dong et al. 2012). These regions can be present independently or together; in the latter case, the Q-rich region is always more $\mathrm{N}$ terminal than the TPAP repeat (Fig. 4B). Adding this domain rich in glutamine or proline, or both, to a cytotoxic NLP or removing it from a cytotoxic NLP that already contains one does not abolish necrosis-inducing activity in transient expression in Nicotiana sp. leaves (Cabral et al. 2012; Dong et al. 2012). This suggests that the necrosis-inducing activity of NLP is independent of the presence or absence of these additional domains.

The proline-rich hydrophilic region has also been described for the noncytotoxic HaNLP9 of $H$. arabidopsidis and was predicted to be O-glycosylated (Cabral et al. 2012). In 27 of the identified Phytophthora NLP, this proline-rich region forms TPAP repeats (Supplementary Fig. S5) that fit the maximum consensus sequence for O-glycosylation (Nishikawa et al. 2010; Wilson et al. 1991). This is also predicted by NetOGlyc3.1 (Julenius et al. 2004), suggesting a high degree of Oglycosylation in these parts of the proteins. The number of TPAP repeats varies from five (Ps_138848, PPTG_08005) up to 14 (Ps_127887). Similar potentially O-glycosylated regions have been described for Phytophthora sojae elicitins (Qutob et al. 2003). The O-glycosylated TPAP-repeat domain has been proposed by Jentoft (1990) to adopt a rigid and extended conformation, like a rod or stick that can penetrate the protective extracellular layer surrounding cells.

Many oomycete NLP have substitutions in the GHRHDWE heptapeptide motif; only $38 \%$ of oomycete NLP contain the fully conserved motif. Examination of the phylogenetic distribution of the oomycete NLP with alterations in the heptapeptide motif revealed that most of these cluster together (Figs. $1 \mathrm{~A}$ and $4 \mathrm{~A}$ ) in one group, which was designated type 1a.

\section{Comparing NLP proteins.}

To gain insight into the similarities and differences among and between the three types of NLP proteins, sequences were aligned and consensus sequences were generated. Based on phylogenetic distribution, we separated the NLP into five distinct groups: fungal/bacterial type 1, oomycete type 1, oomycete type $1 \mathrm{a}$, type 2 , and type 3 . For each type, the conservation of amino-acid residues was determined (details below). Each of these groups is represented by a consensus Weblogo, displaying the conserved residues per group. The Weblogos of the first four groups were aligned to a characterized member of their respective type $\left(\mathrm{NLP}_{\mathrm{Pya}}\right.$ for all type 1 consensus Weblogos and $\mathrm{NLP}_{\mathrm{Pcc}}$ for the type 2 consensus Weblogo) (Fig. 5A). All numbering of type 1 residues is based on the NLP Pya $_{\text {struc- }}$ ture 3GNU (which lacks the SP), and numbering of type 2

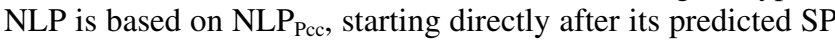
of 22 amino acids. The type 3 consensus Weblogo was found to be too divergent for proper alignment with the other four groups, and is therefore discussed separately below. It was found that the first four groups of NLP differ substantially from each other.
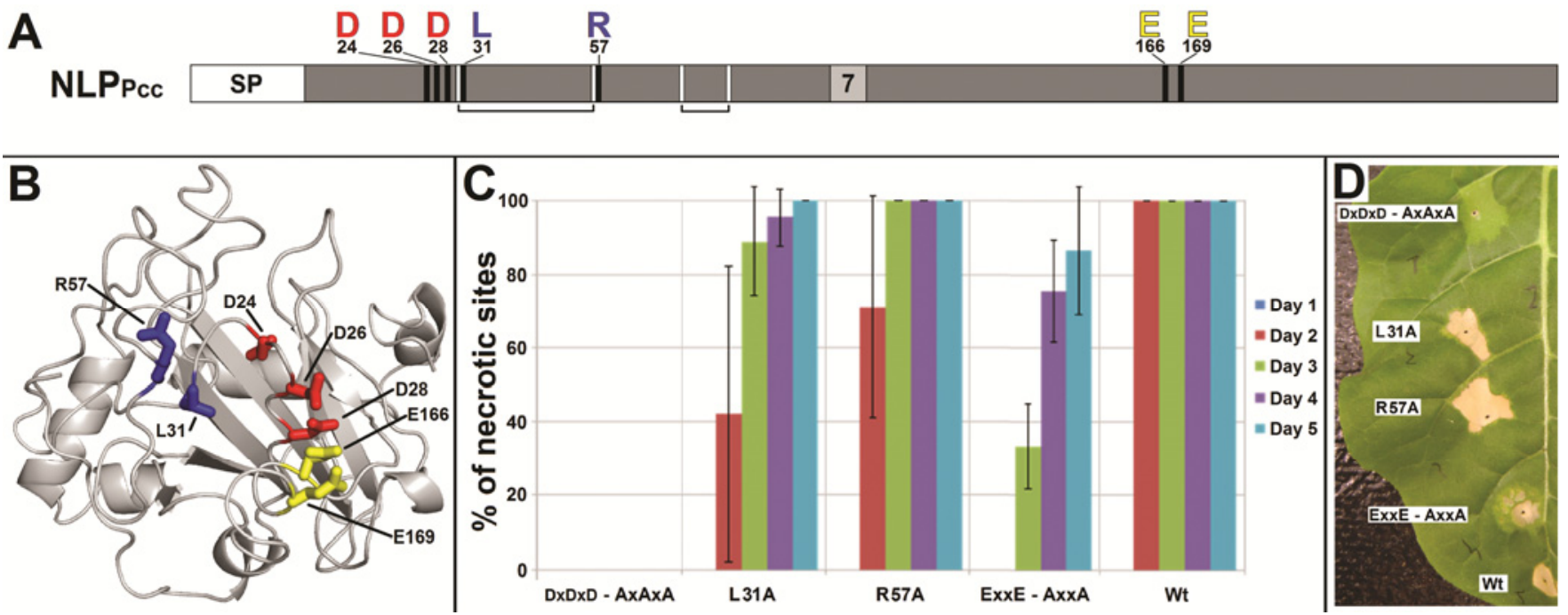

Fig. 6. Effect of alanine substitutions of the putative calcium-binding site and proximate conserved residues of $\mathrm{NLP}_{\mathrm{Pcc}}$ (coordinates based on protein without a signal peptide [SP]). A, Model of NLP $\mathrm{Pcc}_{\text {cc }}$ indicating the location of the mutated residues (black) and the conserved cysteins (white). B, 3D model of

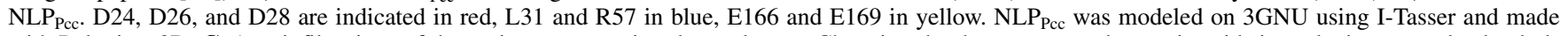
with Polyview 3D. C, Agroinfiltrations of the various mutants in tobacco leaves. Changing the three conserved aspartic acids into alanines completely abolishes necrosis-inducing activity, while L31A and R57A do not affect the cytotoxic activity. Changing the two conserved glutamic acids to alanines results in a reduced activity. Plants were followed to day 10; the last 5 days, no more changes in necrosis were observed. Standard deviation represent $3 \times 15$ plants. D, Changing the two conserved glutamic acids to alanines results in an incomplete, patchy necrosis. Photograph taken at day 5. 


\section{Oomycete type 1a NLP}

lack the acidic cation-binding pocket.

The N-termini, between the SP and the first conserved cysteines (shown in orange in Figure 5) of type 1 and type 2 NLP, differ too much for proper alignment (Fig. 5A). In type 1 NLP, this part contains four highly conserved residues (I3, H5, D6, and V8), which, together with Y40, D44, T49, and S50, are in close proximity to each other in the $3 \mathrm{D}$ structure of NLP $_{\text {Pya }}$ (Supplementary Fig. S6A), forming a region not present or not conserved in type 2 NLP.

When comparing type 1 NLP of fungi and bacteria with those of oomycetes, 12 amino-acid positions were found to be highly conserved in oomycete NLP but less in fungal/bacterial NLP: P10, T18, K22, A24, K26, K28, Q30, S69, S124, D172, Q178, and R184. Except for S124, all these residues are on the surface and are located on one side of the protein (Supplementary Fig. S7). Notably, a number of positively charged amino acids is highly conserved, suggesting these oomycete proteins have a local surface charge different from fungal type 1 NLP. When comparing the cytotoxic fungal/ bacterial and oomycete type 1 NLP to the noncytotoxic oomycete type 1a NLP, several differences are observed. Of the seven amino acids making up the heptapeptide motif, type 1a NLP only show conservation of three (R102, H103, and W105), whereas four others do not (G100, H101, D104, and E106). Of these latter four, three residues (H101, D104, and E106) have been shown to be crucial for necrosis induction in $\mathrm{NLP}_{\mathrm{Pya}}$ and are part of its acidic cation-binding pocket (Ottmann et al. 2009). In each type 1a NLP, one or more of the eight residues making up this pocket (D93, H101, D104, E106, H128, Y151, D/N158, and H159) (Fig. 5B) are substituted. Also, three residues near this pocket (G100, G193, and A195) that are highly conserved in both oomycete type 1 NLP, fungal/bacterial type $1 \mathrm{NLP}$, and type 2 NLP are not conserved in type 1a NLP, suggesting they play a role in cytotoxicity. The same 11 residues are substituted in most Rhizobiaceae NLP and in the noncytotoxic branch of Glomerella/ Colletotrichum NLP in which the heptapeptide was found to have substitutions (Fig. 3, Supplementary Fig. S8). The only residue that is highly conserved in type 1a NLP but not in the other type 1 NLP is D200 (corresponding to K200 in NLP Pya $_{\text {). }}$.

\section{Type 3 NLP contain all cation-binding residues.}

As type 3 NLP share no clear homology with the other two types of NLP, except for the 50 residues surrounding the heptapeptide motif, the rest of the sequence cannot be compared with type 1 and type 2 NLP. Most type 3 NLP (22 of 33) are characterized by having six conserved cysteines corresponding to positions 39, 67, 74, 76, 99, and 270 in Afu5g02100 (Supplementary Fig. S9A). Eleven type 3 NLP do not have cysteine residues corresponding to positions 67 and 74, suggesting that in the other 22 type 3 NLP C67 and C74 form a disulfide bridge. In a single protein (ELR02678 from Geomyces destructans), cysteines 39 and 76 have been substituted for a threonine and a serine, respectively, while the other four cysteines are present, suggesting C39 and C76 form a disulfide bridge as well in the other proteins. The 50-amino-acid region that contains the heptapeptide motif can be aligned with the homologous region from type 1 and type 2 NLP. Strikingly, all residues in this region of $\mathrm{NLP}_{\text {Pya }}$, which make up the acidic cation-binding pocket (D93, G100, H101, D104, E106, and H128) and which are also found in the cytotoxic type $2 \mathrm{NLP}_{\mathrm{Pcc}}$, are highly conserved in type 3 NLP, suggesting that type 3 NLP also bind a cation. The amino-acid sequences $\mathrm{N}$ - and $\mathrm{C}$-terminal of the conserved 50-residue-stretch are very different from those of types 1 and 2 NLP. The arginine and histidine residues (underlined) of the heptapeptide motif (GHHDWE), which are not conserved in type 3 NLP, are not surface exposed but, rather, seem to play a structural role in the NLP ${ }_{\text {Pya }}$ protein.

\section{Type 2 NLP contain a putative calcium-binding motif.}

Based on their amino-acid sequence, type 2 NLP share an overall structure with type 1 NLP, yet there are specific differences. In type $2 \mathrm{NLP}$, we find a strongly conserved $\mathrm{Kx} \underline{\mathrm{VY}} \underline{\mathrm{HK}}$ DxxxTHxxA motif (residues 146 to 162 in NLP $_{\text {Pcc }}$ ), of which the five underlined residues show no conservation in type 1 NLP (Fig. 5A). The corresponding five positions in NLP Pya (S150, K152, S153, T154, and G162) are in close proximity to each other and locate to two antiparallel beta sheets, suggesting they form a positively charged patch.

Besides this region, type 2 NLP contain two other regions that differ strongly from type 1 NLP. Interestingly, several residues in these regions show a high level of conservation in type

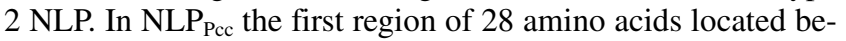
tween the SP and the glycine residue preceding the first conserved cysteine contains six conserved residues (Fig. 5A). A second, nine-amino acid region (of which three are conserved), corresponding to residue E166 to K174 in $\mathrm{NLP}_{\mathrm{Pcc}}$, is predicted to form an additional (surface-exposed) loop and corresponds to a smaller region in $\mathrm{NLP}_{\text {Pya }}$ (between T165 and G168). The 28-amino acid N-terminal region contains a highly conserved DxDxDG motif (residues 24 to 29), which could function as a calcium-binding motif (Rigden and Galperin 2004). The second region (166 to 174 in NLP $_{\text {Pcc }}$ ) contains three conserved residues: E166, E169, and N170. The two corresponding regions in $\mathrm{NLP}_{\text {Pya }}$ (L31-N35 and T165G168) are in close proximity. This suggests the two acidic regions form a binuclear calcium-binding center (Rigden and Galperin 2004). When modeled over the structure of NLP ${ }_{\text {Pya }}$, the putative calcium-binding center of NLP $_{\text {Pcc }}$ is visible as a concentrated patch of negatively charged residues (Fig. 6A and B). Other residues conserved in type 2 but not in type 1 NLP correspond to $\mathrm{NLP}_{\mathrm{Pcc}} \mathrm{L} 31$, L38, N43, R57, R64, E91, and F175.

To investigate whether this negatively charged surface region of $\mathrm{NLP}_{\mathrm{Pcc}}$ is essential for necrosis induction, alanine-substituted versions were created in the conserved DxDxD motif and in residues E166 and E169 as well as conserved residues L31 and R57, which are predicted to be on the surface of the protein, close to the DxDxD motif (Fig. 6B) and conserved in type 2 but not in type 1 NLP (Fig. 5A). Transient expression of these alanine-substituted proteins showed that the DxDxD to AxAxA variant of $\mathrm{NLP}_{\mathrm{Pcc}}$ no longer induces necrosis, while the L31A and R57A variants did not show a marked reduction in the induction of necrosis (Fig. 6C). NLP $\mathrm{Pcc}_{\mathrm{cc}}$ with the substitutions E166xxE169 to A166xxA169 induced necrosis formation, although it was delayed and patchy (Fig. 6D). These data suggest that the conserved acidic residues, predicted to be involved in calcium binding and lacking in type 1 NLP, are important for necrosis induction by type 2 NLP.

\section{NLP have a high number of different potential disulfide bridges.}

While comparing the obtained type 1 and type 2 protein sequences, we noticed several conserved cysteine pairs that could form disulfide bridges. Cysteine residues that are part of disulfide bridges are in close proximity in the 3D structures and are orientated such that their sulfur groups are able to form a covalent bond. When, for a given protein, the crystal structure of a homologous protein is available, disulfide bridges can be predicted based on the occurrence of cysteines and their location in the protein. The distance between the $\alpha$-carbons $(\mathrm{C}$ $\alpha$ ) of the two cysteines provides a measure of the proximity in the protein structure, whereas the distance between the $\beta$ car- 
bons $(C \beta)$ provides a proxy for the distance of the corresponding sulfur groups $(\mathrm{S})$ (Fig. 7A).

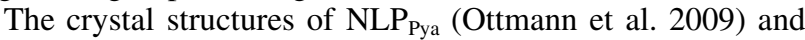
MpNEP2 (Zaparoli et al. 2011), show that the two strongly conserved cysteines, which are present in both type 1 and type 2 NLP, form a disulfide bridge, which we refer to as "bridge A." A second pair of conserved cysteines is found in most type 2 NLP. As no type 2 NLP structure is available, we interrogated the positions (outlined below) of the corresponding resi-

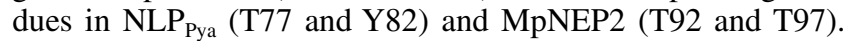
We concluded that these residues are in close proximity, making it likely that the corresponding cysteines in type 2 NLP form a second disulfide bridge (Fig. 7), which we refer to as "bridge B."

A search in our collected type 1 and type 2 NLP sequences resulted in more than 100 sequences containing two or more extra cysteine residues in addition to the cysteines making up one or both bridge $\mathrm{A}$ and $\mathrm{B}$. To predict if the additional cysteine residues are located in sufficient proximity to form a disulfide bridge, we located the corresponding residues for each of them in the structures of $\mathrm{NLP}_{\mathrm{Pya}}(3 \mathrm{GNU})$ and MpNEP2 (3ST1) (Fig. 7B). Based on the measured distances of cysteine residues, 12 additional potential disulfide bridges in the NLP family were identified in types 1 and 2 NLP (Fig. 7C). The occurrence of these extra disulfide bridges in NLP is indicated in the phylogenetic protein trees (Figs. 2, 3, and 4). Of these 12 identified additional disulfide bridges, only three (bridges 6, 7, and 9) are occurring in more than 10 NLP. Bridge 6 is found in a branch of oomycete NLP containing both cytotoxic (PsNLP59; Dong et al.
2012) and noncytotoxic (HaNLP3, Cabral et al. 2012) NLP. Bridge 7 is found in a branch of fungal type 1 NLP. In one of the NLP containing this bridge (BcNEP2; Arenas et al. 2010), this bridge was disrupted by amino-acid substitutions without any change in necrosis-inducing activity. Bridge 9 was identified in 16 fungal type 2 NLP and in one unrelated bacterial type 2 NLP, suggesting this bridge has evolved twice independently.

In three additional cases, one of the cysteines potentially making up the disulfide bridge is located in a region just $\mathrm{N}$ or $\mathrm{C}$ terminal of the part of the proteins that is present in the crystal structures of $\mathrm{NLP}_{\mathrm{Pya}}$ and MpNEP2, thus not allowing exact distance measurements of $\mathrm{C} \alpha$ and $\mathrm{C} \beta$ of their corresponding residues. Of these, one cysteine pair is present in 14 NLP of species in the genus Glomerella/Colletotrichum, another in four NLP of $H$. arabidopsidis (HaNLP1, 2, 4, and 6) and in a Peronospora tabacina homolog (Supplementary Fig. S10), and a third in HaNLP2. Including these three additional disulfide bridges, 17 potential disulfide bridges are found in the NLP family. Previously, fifteen distinct disulfide bridges were found in the family of $\beta$-glucanases that comprises cellulases, glucanases, xylanases, glucuronidases, and mannases encompassing 14 Pfam domains (Thangudu et al. 2008). In contrast, the NLP family only has a single Pfam domain and has, to our knowledge, the highest number of distinct potential disulfide bridges found in any protein family.

\section{Functionality of disulfide bridges in the NLP.}

Formation of bridge A seems essential for necrosis induction, as PpNPP1 of Phytophthora parasitica (Fellbrich et al.

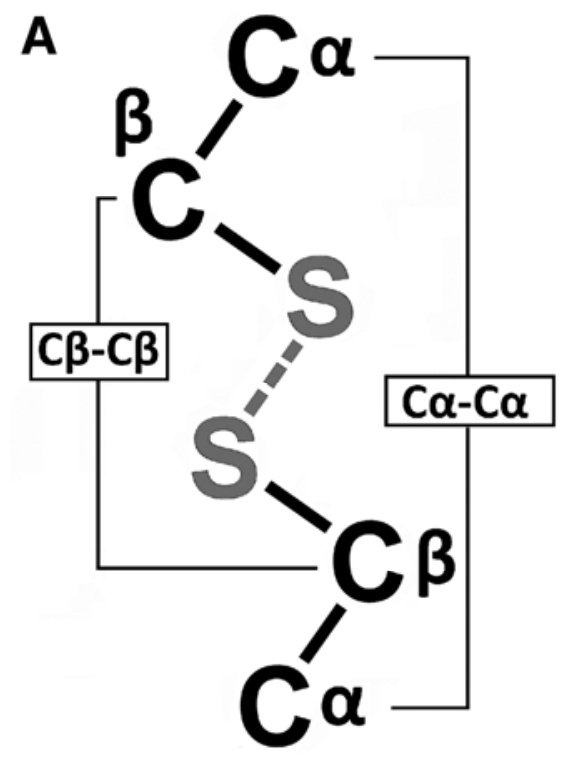

\begin{tabular}{|c|c|c|c|c|c|c|c|}
\hline \multirow[b]{3}{*}{ Bridge } & \multirow{3}{*}{$\begin{array}{c}\text { Position } \\
\text { Residue nr. }\end{array}$} & \multicolumn{4}{|c|}{ Distance (Ångström) } & & \\
\hline & & \multicolumn{2}{|c|}{ 3GNU } & \multicolumn{2}{|c|}{ 3ST1 } & \multicolumn{2}{|l|}{ Occurence } \\
\hline & & $\mathrm{C} \alpha-\mathrm{C} \alpha$ & $C \beta-C \beta$ & $\mathrm{C} \alpha-\mathrm{C} \alpha$ & $C \beta-C \beta$ & Nr. of proteins & Type \\
\hline A & $37-63$ & 4,93 & 3,52 & 5,01 & 3,64 & 524 & $1 \& 2$ \\
\hline B & $77-82$ & 4,36 & 5,61 & 5,28 & 6,06 & 119 & 2 \\
\hline (1) & $56-98$ & 3,81 & $5,09^{*}$ & 4,77 & $5,42^{*}$ & 2 & 2 \\
\hline 2 & $58-97$ & 4,12 & 4,62 & 6,39 & 7,38 & 2 & 2 \\
\hline 3 & 69-171 & 5,27 & 3,98 & 5,17 & 3,45 & 1 & 2 \\
\hline 4 & $82-115$ & 5,87 & 4,55 & 6,13 & 6,75 & 7 & 1 \\
\hline (5) & 83-111 & 4,76 & 5,13 & 6,4 & 5,52 & 1 & 1 \\
\hline 6 & $123-137$ & 6,01 & 5,32 & 7,22 & 5,36 & 20 & 1 \\
\hline 7 & $124-133$ & 4,45 & 5,16 & 4,44 & 5,11 & 43 & 1 \\
\hline 8 & $124-210$ & 5,66 & 3,82 & 5,41 & $3,88^{*}$ & 3 & 2 \\
\hline 9 & $127-160$ & 4,64 & 3,91 & 4,57 & 4,01 & 17 & 2 \\
\hline (10) & $134-138$ & 6,06 & 4,86 & 9,67 & $7,50^{*}$ & 1 & 1 \\
\hline (11) & $141-163$ & 5,96 & 4,1 & 6,52 & 5,08 & 2 & 1 \\
\hline (12) & $197-200$ & 6,18 & 4,52 & 5,51 & 4,32 & 1 & 1 \\
\hline
\end{tabular}

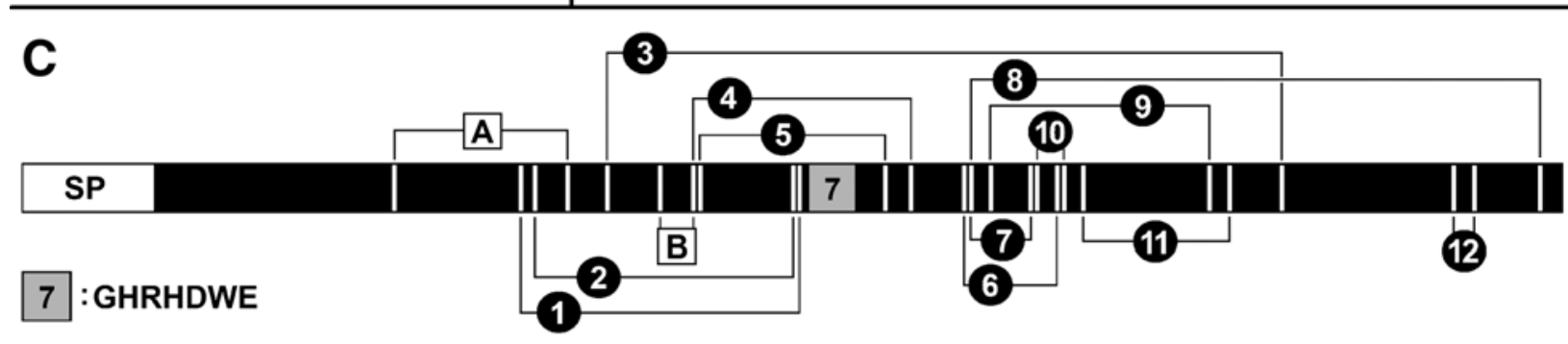

Fig. 7. Occurrence of possible disulfide bridges in type 1 and type 2 Nep1-like proteins (NLP). A, Disulfide bridges in proteins consist of two cysteine residues, each possessing an $\alpha$ carbon, a $\beta$ carbon, and a sulfur. Distances of these atoms between the cysteines are an indication of the disulfide-forming capability of two closely positioned cysteines. $\mathbf{B}$, Distances of the pairs of $\alpha$ carbons and pairs of $\beta$ carbon of the predicted disulfide bridges in NLP Pya (3GNU) and MpNEP2 (3ST1). Residue numbering is based on PDB:3GNU. Asterisks (*) indicates a glycine (lacking a $\beta$ carbon) located at one of the positions of the possible disulfide bridge. In these cases the glycine was replaced in the structure by an alanine, using I-Tasser, and the position of the alanine $\beta$ carbon was used. $\mathbf{C}$, Position of the predicted disulfide bridges in different NLP plotted on a line drawing of NLP $P_{\text {Pya }}$. 
2002), BcNEP1, and BcNEP2 of Botrytis cinerea (Arenas et al. 2010) do not induce necrosis when one of these cysteines is substituted. To test the requirement of the strongly conserved bridge $B$ in type 2 NLP for induction of necrosis, mutant proteins of $\mathrm{NLP}_{\mathrm{Pcc}}$ were created in which bridge $\mathrm{A}$, bridge $\mathrm{B}$, or both are disrupted by substituting one of the cysteines by a serine (Fig. 8A). Disruption of bridge A in both PsojNIP and $\mathrm{NLP}_{\mathrm{Pcc}}$ led to a complete loss of necrosis induction (Fig. 8B), while disruption of bridge $\mathrm{B}$ in $\mathrm{NLP}_{\mathrm{Pcc}}$ did not affect its cytotoxicity. Also, simultaneous disruption of both bridges $\mathrm{A}$ and $\mathrm{B}$ led to a complete loss of necrosis induction. These results suggests that bridge $\mathrm{B}$, although highly conserved in type 2 NLP, is not essential for necrosis induction but has possibly evolved to enhance protein stability.

\section{Necrosis induction by NLP targeted}

\section{to different plant cell compartments.}

Experiments by Qutob and associates $(2002,2006)$ indicated that the induction of cell death by transient expression of PsojNIP in plant cells requires the presence of a SP, suggesting that NLP require an as-yet-unidentified target located on the extracellular side of dicot plasma membranes. However, it is also possible that disulfide bridge A, which is required for necrosis induction by both PsojNIP and NLP Pcc (Fig. 8B), may not be created when the proteins are produced in the plant cell cytoplasm, an environment that does not support the effective formation of disulfide bonds (Raina and Missiakas 1997). To test if PsojNIP and NLP $P_{\text {Pcc }}$ would induce cell death when produced in the endoplasmic reticulum (ER) (in which cysteine bonds are formed [Raina and Missiakas 1997]), we added a C-terminal KDEL sequence to these NLP. Proteins with a C-terminal KDEL sequence that enter the ER are unable to exit it. Leakage from the ER is unlikely, as protein retention in the ER is not significantly impaired by an increased concentration of KDEL-carrying proteins (Denecke et al. 1992). The KDEL sequence is highly specific; the chemically similar sequence KDDL does not function as an ER retention signal (Denecke et al. 1992) and can, therefore, be used as a control. As shown in Figure 9, NLP $\mathrm{Pcc}_{\mathrm{Pc}}$, with a Cterminal KDEL sequence, does not induce any visible necrosis, while its control, KDDL, gives wild-type levels of necrosis. NLP $\mathrm{P}_{\mathrm{Pcc}}$ lacking its SP also does not induce any necrosis. In contrast, PsojNIP with a C-terminal KDEL sequence has a one-day delay in onset of necrosis, yet reaches full necrosis at day 4. To our surprise, PsojNIP, lacking a SP, still caused necrosis, although delayed compared with the wild-type protein, while $\mathrm{NLP}_{\mathrm{Pcc}}$ without a SP did not induce any necrosis.

Although transient expression in planta is a highly artificial system and may not mimic the natural situation, the difference between type 1 PsojNIP and type 2 NLP $_{P c c}$ is remarkable and may suggest these proteins do not have the same mode of action, although they both induce a similar necrotic response and type 1 NLP can restore virulence of the Pectobacterium carotovorum $\mathrm{NLP}_{\mathrm{Pcc}}$ mutant (Ottmann et al. 2009). A difference is also observed when the wild-type proteins (with SP)

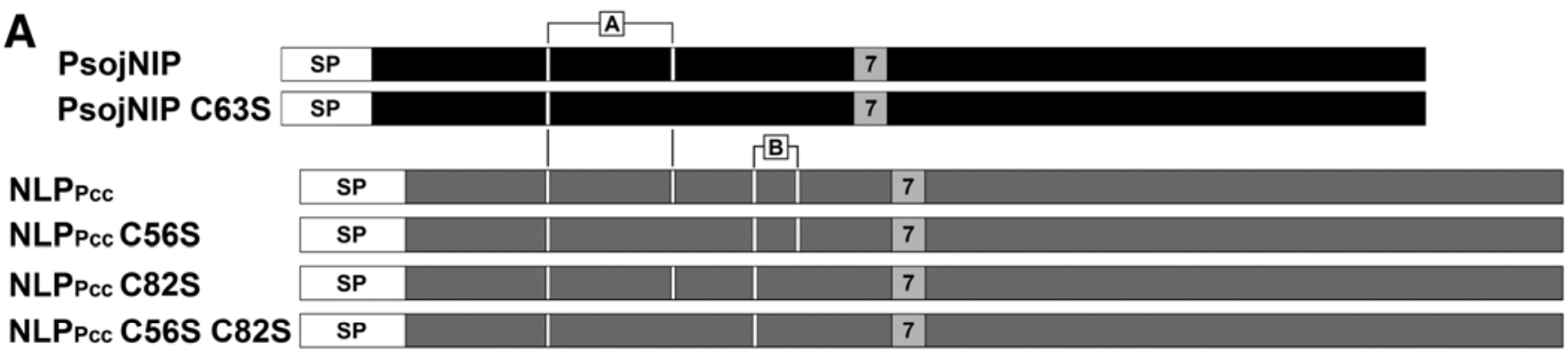

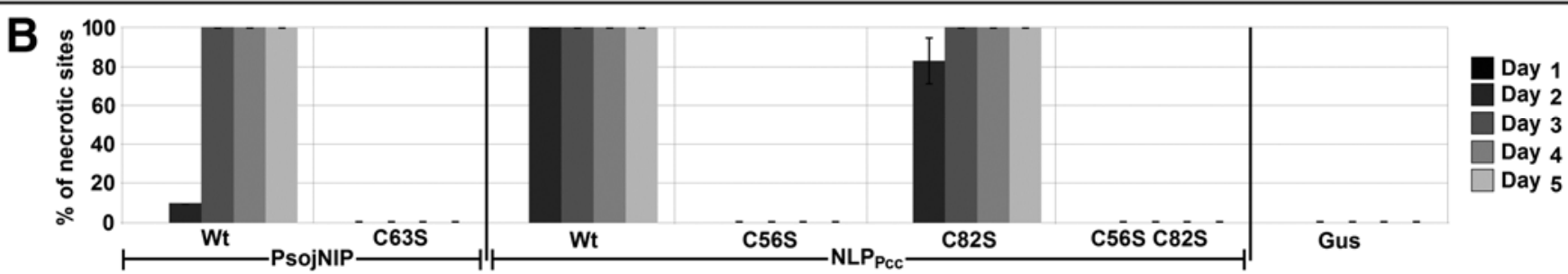

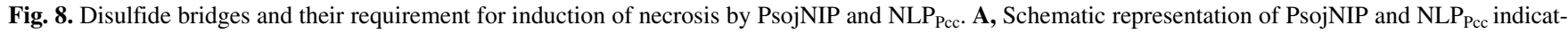
ing the different disulfide bridges. $\mathbf{B}$, Disulfide bridge $\mathrm{A}$ is required for necrosis-inducing activity in both PsojNIP and NLP $\mathrm{P}_{\mathrm{Pcc}}$, while disulfide bridge $\mathrm{B}$ is not required for necrosis-inducing activity in $\mathrm{NLP}_{\mathrm{Pcc}}$. Transient assays in tobacco, using agroinfiltration. As a negative control, $\beta$-Glucuronidase (GUS) was expressed. Plants were followed to day 10 ; the last 5 days, no more changes in necrosis were observed. Standard deviation shown is from $3 \times 15$ plants.

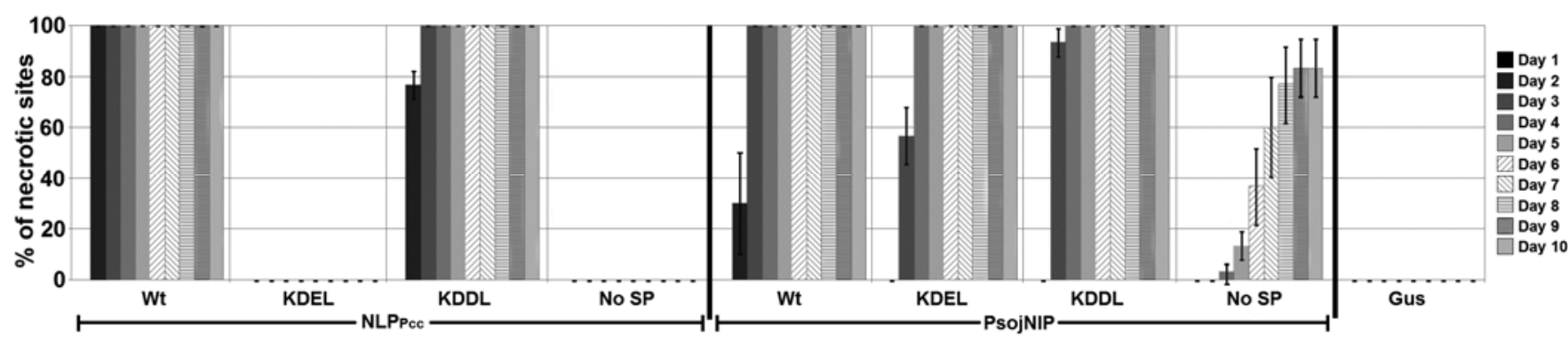

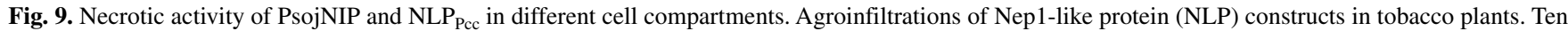
plant were infiltrated and the experiment was repeated three times. As a negative control, $\beta$-Glucuronidase (GUS) was expressed. 
are tested by transient expression; NLP $_{\text {Pcc }}$ requires about one less day than PsojNIP to induce necrosis (Figs. 8 and 9).

Concluding remarks on the versatile NLP protein family.

We have defined four different groups of NLP belonging to three phylogenetic types, which are schematically represented in Figure 10. Type 1 NLP are characterized by the presence of (at least) a single conserved disulfide bridge (C-C), an acidic cation-binding pocket, and an exposed region, which are all known to be required for cytotoxicity (Cabral et al. 2012; Fellbrich et al. 2002; Ottmann et al. 2009). Type 1a NLP, which diverged from type 1 NLP and are strongly expanded in oomycetes, are similar to type 1 NLP yet have substitutions in the acidic cation-binding pocket that is conserved in all other NLP types. Type 2 NLP are not only distinguished by a second conserved disulfide bridge but, more importantly, by the presence of a putative calcium-binding domain, equivalent to the exposed region found in types 1 and $1 \mathrm{a}$ NLP and that is required for the induction of necrosis. In contrast to type 1 NLP, no type 2 equivalent of the type 1a NLP (i.e., type 2 NLP that lack the acidic cation-binding pocket) were identified. Our knowledge of type 3 NLP is still very limited. Based on the presence of conserved cysteines, we predict that most type 3 NLP contain three disulfide bridges. In addition, the strong conservation of residues that make up the cation-binding pocket in types 1 and 2 NLP suggests that type 3 NLP also contain a similar pocket. The $\mathrm{N}$ - and C-terminal parts of type 3 NLP are very different from type 1 and type 2 NLP, precluding any other structure-based predictions.

In the described research, a large number of newly identified NLP sequences from a broad range of diverse organisms was studied to provide insight into the evolution and functions of these remarkable proteins. Phylogenetic analysis revealed three distinct types (and an additional subtype) of NLP, in contrast to the previously described two NLP types (Gijzen and Nürnberger 2006). While type 1 NLP are found almost exclusively in plant-associated microbes, type 2 and type 3 NLP occur in microorganisms with different lifestyles. As only Ascomycete fungi have all three NLP types, it is tempting to speculate that NLP find their origin in this group of fungi. In oomycetes, only type 1 NLP occur, which have strongly expanded in this group of plant pathogens. In addition, oomycetes have evolved NLP with extensive N-terminal modifications, of which the role is not clear, yet these additional domains do not interfere with the induction of necrosis. The oomycete type 1a NLP have extensive substitutions in the acidic cation-binding pocket and have lost the ability to induce necrosis. Similar substitutions in the acidic cation-binding pocket were also found in type 1 NLP of members of the plant symbiotic group of Rhizobiaceae and in the hemibiotrophic fungi of the genus Colletotrichum, suggesting these have evolved at least three times independently. This suggests that noncytotoxic type 1 NLP lacking the cation-binding pocket serve an as-of-yet unknown function that is not related to the induction of necrosis.

Our multifaceted analysis showed that the NLP domain is highly versatile and has been the basis for at least four different groups of NLP. These proteins are very widespread, both phylogenetically and among microbial lifestyles. In many species, more than one of these groups co-occur, suggesting a different function for each of them. This hypothesis is supported by the finding that necrosis-inducing types 1 and 2 NLP do not only contain different conserved motifs but, also, exhibit different responses when transiently expressed in planta. All these distinctions should be taken into account and may be of great help in deciphering the molecular mechanisms and biological roles of the members of this fascinating protein family.

\section{MATERIALS AND METHODS}

\section{Finding NLP genes.}

Databases (National Center for Biotechnology Information, FungiDB, Joint Genome Institute, and Endophyte) were searched for NLP homologs using BlastP with PsojNIP (type 1), $\mathrm{NLP}_{\text {Pcc }}$ (type 2), and Afu5g02100 (type 3) as queries. Only for searching the Endophyte database, TBlastN was used, because BlastP was not available. Only full-length protein sequences were included in the analysis.

\section{Construction of phylogenetic trees.}

Neighbor-joining trees were created by aligning the NLP protein sequences with MEGA5 (ClustalW, standard setting, 1,000 bootstraps). Maximum likelihood trees were created by aligning individual NLP types using mafft (LINSi, GINSi, and EINSi) and muscle. Positions that were present in fewer than $80 \%$ of the aligned proteins were removed from the alignment, using a customized script. In both methods, all present SP were predicted by SignalP4.1 and were removed before analysis, as these can share similarity not based on ancestry but on physico-chemical constraints (Wong et al. 2010). The best scoring alignment (mafft-LINSi) was chosen based on the quality indications from normd and mumsa. Phylogenetic trees were constructed using phyml (v3.0, WAG model of aminoacid substitution, number of substitution rate categories 4 , estimated gamma distribution parameters, and estimated proportion of invariable sites), and the robustness was assessed with 1,008 bootstrap replicates. The best-fitting amino-acid substitution model and parameter settings were chosen based on

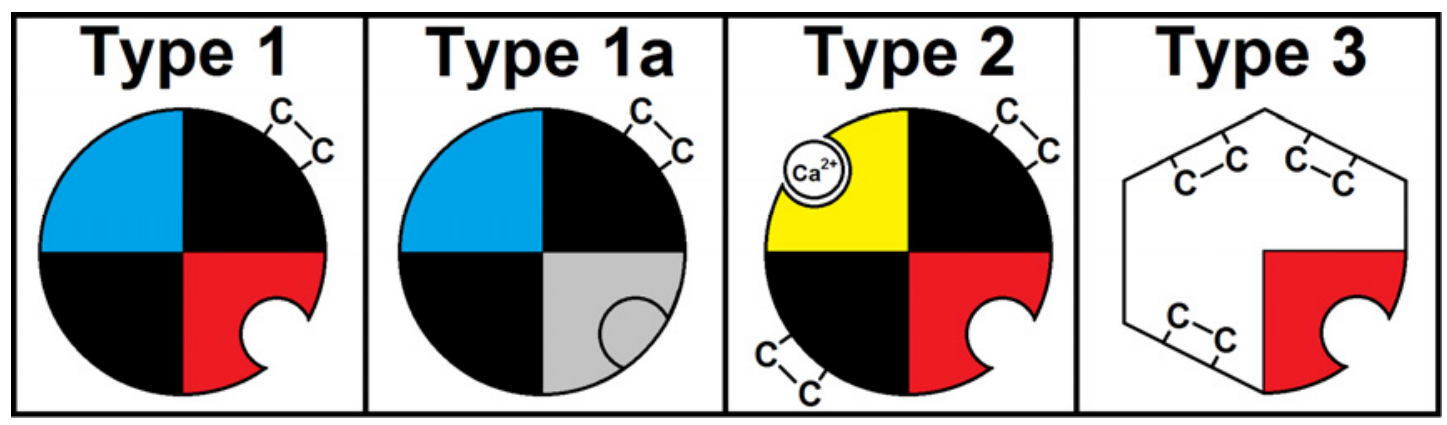

Fig. 10. Schematic representation of the different Nep1-like proteins (NLP). Types 1, 1a, and 2 share a comparable protein structure, while the overall structure of type 3 NLP is likely different. Types 1,2, and likely 3 , share a conserved cation-binding pocket (red) that is required for cytotoxicity. This pocket is lacking in type 1a NLP and is probably the cause of their noncytotoxicity. Type 1 and type 1a NLP share a region (blue), part of which is involved in cytotoxicity (Cabral et al. 2012), yet which is also highly conserved in noncytotoxic type 1a NLP. On this location in the protein, type 2 NLP have a predicted calcium-binding site (yellow) not found in the other types of NLP, yet required for their cytotoxicity. 
ProtTest. The created tree files were uploaded in FigTree v1.4.0 to produce the tree image.

\section{Consensus Weblogos.}

The NLP amino-acid sequences (without SP) were aligned per type and group using ClustalW. The generated alignment file was used in Consurf without a tree file, using the model NLP $_{\text {Pya }}$ (PDB:3GNU) for the type 1 NLP. For the type 2 NLP, $\mathrm{NLP}_{\mathrm{Pcc}}$ (without SP) was modeled on $3 \mathrm{GNU}$, using I-Tasser. For type 3 NLP, Afu5g02100 (without its 20-amino acid SP) was used as a model without PDB file, because Afu5g02100 is too divergent from $3 \mathrm{GNU}$ and does not have any related published structural homologs. For types 1 and 2 NLP, all positions scoring a conservation of 8 or 9 in Consurf were selected. Positions scoring below 8 or 9 in the selected type and group yet scoring 8 or 9 in the other group were also selected for the Weblogo, to show this homologous position is less conserved. For the type 3 NLP, all positions scoring 7, 8, or 9 in Consurf were used, as fewer sequences are available. Weblogos were created with Weblogo. Colors were set at KRH: green, DE: blue, ST: red, VLAGI: yellow (RGB:FFFF00), NQ: purple, and $\mathrm{C}$ : orange. Alignment gaps of the Weblogos were manually corrected using structural information from $\mathrm{NLP}_{\mathrm{Pya}}$ and the modeled NLP $\mathrm{Pcc}_{\text {. }}$

\section{Constructs.}

Constructs were made by cloning the original genes (PsojNIP, $\left.\mathrm{NLP}_{\mathrm{Pcc}}\right)$ into $\mathrm{pENTRY/D-TOPO}$ vector using Gateway cloning (Invitrogen) and were subcloned into pB7WG2 (Karimi et al. 2002) or pFAST (Shimada et al. 2010), which both have a 35S promoter to express the gene in planta. All modifications (point mutations, removal of SP, adding KDEL and KDDL) were done according to the Phusion site-directed mutagenesis protocol (Finnzymes). All primers used are listed in Supplementary Table S2.

\section{Agroinfiltrations in tobacco.}

Five- to six-week old tobacco plants (Nicotiana tabacum cv. Samsun) were grown at short day conditions (10-h light, 14-h darkness) at $21^{\circ} \mathrm{C}$ and $70 \%$ humidity. Agrobacterium tumefaciens $(\mathrm{C} 58 \mathrm{C} 1)(2 \mathrm{ml})$ was grown overnight with the appropriate antibiotics, was spun down ( $1 \mathrm{~min}$ at full speed), and was resuspended in infiltration medium $\left(10 \mathrm{mM} \mathrm{MgCl}_{2}, 0.1 \mathrm{mM}\right.$ morpholineethanesulfonic acid, $\mathrm{pH}$ 5.7) to an optical density at $600 \mathrm{~nm}$ of 0.8 . Acetosyringone was added $(100 \mu \mathrm{M}$ final concentration) and bacteria were incubated for 3 to $4 \mathrm{~h}$ at room temperature. Infiltration in tobacco leaves was performed by puncturing a small hole in the leaf and infiltrating the bacterial suspension with a needleless 1-ml syringe. The infiltrated areas were scored for necrosis 10 consecutive days after infiltration.

\section{ACKNOWLEDGMENTS}

Thanks to M. F. Seidl for assisting in phylogenetic analyses, M. Pirhonen for providing us with the $N L P_{P c c}$ gene, and P. Santhanam, B. Thomma, and C. Wang for sharing expression data.

\section{LITERATURE CITED}

Arenas, Y. C., Kalkman, E. R. I. C., Schouten, A., Dieho, M., Vredenbregt, P., Uwumukiza, B., Osés Ruiz, M., and van Kan, J. A. L. 2010. Functional analysis and mode of action of phytotoxic Nep1-like proteins of Botrytis cinerea. Physiol. Mol. Plant Pathol, 74:376-386.

Bae, H., Bowers, J. H., Tooley, P. W., and Bailey, B. A. 2005. NEP1 orthologs encoding necrosis and ethylene inducing proteins exist as a multigene family in Phytophthora megakarya, causal agent of black pod disease on cacao. Mycol. Res. 109:1373-1385.
Bae, H., Kim, M. S., Sicher, R. C., Bae, H., and Bailey, B. A. 2006. Necrosis- and ethylene-inducing peptide from Fusarium oxysporum induces a complex cascade of transcripts associated with signal transduction and cell death in Arabidopsis. Plant Physiol. 141:1056-1067.

Bailey, B. A. 1995. Purification of a protein from culture filtrates of Fusarium oxysporum that induces ethylene and necrosis in leaves of Erythroxylum coca. Phytopathology 85:1250-5.

Bashi, Z. D., Hegedus, D. D., Buchwaldt, L., Rimmer, S. R., and Borhan, M. H. 2010. Expression and regulation of Sclerotinia sclerotiorum necrosis and ethylene-inducing peptides (NEPs). Mol. Plant Pathol. 11:43-53.

Boller, T., and Felix, G. 2009. A renaissance of elicitors: perception of microbe-associated molecular patterns and danger signals by patternrecognition receptors. Ann. Rev. Plant Biol. 60:379-406.

Cabral, A., Oome, S., Sander, N., Küfner, I., Nürnberger, T., and Van den Ackerveken, G. 2012. Nontoxic Nep1-like proteins of the downy mildew pathogen Hyaloperonospora arabidopsidis: Repression of necrosis-inducing activity by a surface-exposed region. Mol. Plant-Microbe Interact. 25:697-708

Crous, P. W., Schoch, C. L., Hyde, K. D., Wood, A. R., Gueidan, C., de Hoog, G. S., and Groenewald, J. Z. 2009. Phylogenetic lineages in the Capnodiales. Stud. Mycol. 64, 17-47.

Denecke, J., de Rycke, R., and Botterman, J. 1992. Plant and mammalian sorting signals for protein retention in the endoplasmic reticulum contain a conserved epitope. EMBO (Eur. Mol. Biol. Organ.) J. 11:2345255.

Dong, S., Kong, G., Qutob, D., Yu, X., Tang, J., Kang, J., Dai, T., Wang, H., Gijzen, M., and Wang, Y. 2012. The NLP toxin family in Phytophthora sojae includes rapidly evolving groups that lack necrosis-inducing activity. Mol. Plant-Microbe Interact. 25:896-909.

Earl, A. M., Losick, R., and Kolter, R. 2008. Ecology and genomics of Bacillus subtilis. Trends Microbiol. 16:269-275.

Fellbrich, G., Romanski, A., Varet, A., Blume, B., Brunner, F., Engelhardt, S., Felix, G., Kemmerling, B., Krzymowska, M., and Nürnberger, T. 2002. NPP1, a Phytophthora-associated trigger of plant defense in parsley and Arabidopsis. Plant J. 32:375-390.

Gao, Q., Jin, K., Ying, S., Zhang, Y., Xiao, G., Shang, Y.,Duan, Z., Hu, X., Xie, X., Zhou, G., Peng, G., Luo, Z., Huang, W., Wang, B., Fang, W., Wang, S., Zhong, Y., Ma, L., St. Leger, R. J., Zhao, G., Pei, Y., Feng, M., Xia, Y., and Wang, C. 2011. Genome sequencing and comparative transcriptomics of the model entomopathogenic fungi Metarhizium anisopliae and M. acridum. PLoS Genet. 7:e1001264. Published online.

Garcia, O, Macedo, J. A. N., Tiburcio, R., Zaparoli, G., Rincones, J., Bittencourt, L. M. C. 2007. Characterization of necrosis and ethyleneinducing proteins (NEP) in the basidiomycete Moniliophthora perniciosa, the causal agent of witches' broom in Theobroma cacao. Mycol. Res. 111:443-455.

Gijzen, M., and Nürnberger, T. 2006. Nep1-like proteins from plant pathogens: Recruitment and diversification of the NPP1 domain across taxa. Phytochemistry 67:1800-1807.

Hauser, E., Kämpfer, P., and Busse, H. 2004). Pseudomonas psychrotolerans sp. nov. Int. J. Syst. Evol. Microbiol. 54(Pt 5):1633-1637.

Jensen, G. B., Hansen, B. M., Eilenberg, J., and Mahillon, J. 2003. The hidden lifestyles of Bacillus cereus and relatives. Environmental Microbiology, 5:631-640.

Jentoft, N. 1990. Why are proteins O-glycosylated? Trends in Biochemical Sciences 15:291-4.

Julenius, K. 2004. Prediction, conservation analysis, and structural characterization of mammalian mucin-type O-glycosylation sites. Glycobiology 15:153-64.

Kanneganti, T.-D., Huitema, E., Cakir, C., and Kamoun, S. 2006. Synergistic interactions of the plant cell death pathways induced by Phytophthora infestans Nep1-like protein PiNPP1.1 and INF1 elicitin. Mol. Plant-Microbe Interact. 19:854-863.

Karimi, M., Inzé, D., and Depicker, A. 2002. GATEWAY vectors for Agrobacterium-mediated plant transformation. Trends Plant Sci. 7:193-195.

Kemen, E., Gardiner, A., Schultz-Larsen, T., Kemen, A. C., Balmuth, A. L., Robert-Seilaniantz, A., Bailey, K., Holub, E., Studholme, D. J., MacLean, D., and Jones, J. D. G. 2011. Gene gain and loss during evolution of obligate parasitism in the white rust pathogen of Arabidopsis thaliana. PLoS Biol.9:e1001094. Published online.

Kleemann, J., Rincon-Rivera, L. J., Takahara, H., Neumann, U., van Themaat, E. V., van der Does, H. C., Hacquard, S., Stüber, K., Will, I., Schmalenbach, W., Schmelzer, E., and O'Connell, R. J. 2012. Sequential delivery of host-induced virulence effectors by appressoria and intracellular hyphae of the phytopathogen Colletotrichum higginsianum. PLoS Pathog. 8:e1002643. Published online.

Küfner, I., Ottmann, C., Oecking, C., and Nürnberger, T. 2009. Cytolytic toxins as triggers of plant immune response. Plant Signal. Behav.4:977979. 
Links, M. G., Holub, E., Jiang, R. H., Sharpe, A. G., Hegedus, D., Beynon, E., Sillito, D., Clarke, W. E., Uzuhashi, S., and Borhan, M. H. 2011. De novo sequence assembly of Albugo candida reveals a small genome relative to other biotrophic oomycetes. BMC Genomics, 12:503. Published online.

Mattinen, L., Tshuikina, M., Mäe, A., and Pirhonen, M. 2004. Identification and characterization of Nip, necrosis-inducing virulence protein of Erwinia carotovora subsp. carotovora. Mol. Plant-Microbe Interact. 17:1366-1375.

Motteram, J., Küfner, I., Deller, S., Brunner, F., Hammond-Kosack, K. E., Nürnberger, T., and Rudd, J. J. 2009. Molecular characterization and functional analysis of MgNLP, the sole NPP1 domain-containing protein, from the fungal wheat leaf pathogen Mycosphaerella graminicola. Mol. Plant-Microbe Interact. 22:790-799.

Nishikawa, I., Nakajima, Y., Ito, M., Fukuchi, S., Homma, K., and Nishikawa, K. 2010. Computational prediction of O-linked glycosylation sites that preferentially map on intrinsically disordered regions of extracellular proteins. Int. J. Mol. Sci. 11:4991-5008.

Ottmann, C., Luberacki, B., Küfner, I., Koch, W., Brunner, F., Weyand, M., Mattinen, L., Pirhonen, M., Anderluh, G., Seitz, H. U., Nürnberger, T., and Oecking, C. 2009. A common toxin fold mediates microbial attack and plant defense. Proc. Natl. Acad. Sci. U.S.A. 106:10359-10364.

Pemberton, C. L., and Salmond, G. P. 2004. The Nep1-like proteins-A growing family of microbial elicitors of plant necrosis. Mol. Plant Pathol. 5:353-359.

Pemberton, C. L., Whitehead, N. A., Sebaihia, M., Bell, K. S., Hyman, L. J., Harris, S. J., Matlin, A. J., Robson, N. D., J. Birch, P. R., Carr, J. P., Toth, I. K., and Salmond, G. P. C. 2005. Novel quorum-sensing-controlled genes in Erwinia carotovora subsp. carotovora: Identification of a fungal elicitor homologue in a soft-rotting bacterium. Mol. PlantMicrobe Interact. 18:343-353.

Qutob, D., Kamoun, S., and Gijzen, M. 2002. Expression of a Phytophthora sojae necrosis-inducing protein occurs during transition from biotrophy to necrotrophy. Plant J. 32, 361-373.

Qutob, D., Huitema, E., Gijzen, M., and Kamoun, S. 2003. Variation in structure and activity among elicitins from Phytophthora sojae. Mol. Plant Pathol. 4:119-124.

Qutob, D., Kemmerling, B., Brunner, F., Küfner, I., Engelhardt, S., Gust, A. A., Luberacki, B., Seitz, H.U., Stahl, D., Rauhut, T., Glawischnig, E., Schween, G., Lacombe, B., Watanabe, N., Lam, E., Schlichting, R., Scheel, D., Nau, K., Dodt, G., Hubert, D., Gijzen, M., and Nürnberger, T. 2006. Phytotoxicity and innate immune responses induced by Nep1like proteins. Plant Cell 18:3721-44.

Raina, S., and Missiakas, D. 1997. Making and breaking disulfide bonds. Ann. Rev. Microbiol. 51:179-202.

Richards, T. A., Soanes, D. M., Jones, M. D., Vasieva, O., Leonard, G., Paszkiewicz, K., Foster, P. G., Hall, N., and Talbot, N. J. 2011. Horizontal gene transfer facilitated the evolution of plant parasitic mechanisms in the oomycetes. Proc. Natl. Acad. Sci. U.S.A. 108:15258-63.

Rigden, D. J., and Galperin, M. Y. 2004. The DxDxDG motif for calcium binding: Multiple structural contexts and implications for evolution. J. Mol. Biol. 343:971-84.

Santhanam, P., van Esse, H. P., Albert, I., Faino, L., Nürnberger, T., and Thomma, B. P. H. J. 2013. Evidence for functional diversification within a fungal NEP1-like protein family. Mol. Plant-Microbe Interact. 26:278-286.

O de Santos, E., Alves, N., Dias, G. M., Mazotto, A. M., Vermelho, A., Vora, G. J., Wilson, B., Beltran, V. H., Bourne, D. G., Le Roux, F., and Thompson, F. L. 2011. Genomic and proteomic analyses of the coral pathogen Vibrio coralliilyticus reveal a diverse virulence repertoire. The International Soc. Microb. Ecol. J. 5:1471-1483.

Schouten, A., van Baarlen, P., and van Kan, J. A. 2008. Phytotoxic Nep1like proteins from the necrotrophic fungus Botrytis cinerea associate with membranes and the nucleus of plant cells. New Phytol. 177:493-505.

Shimada, T. L., Shimada, T., and Hara-Nishimura, I. 2010. A rapid and non-destructive screenable marker, FAST, for identifying transformed seeds of. Plant J. 61:519-28.
Staats, M., van Baarlen, P., Schouten, A., and van Kan, J. A. 2007. Functional analysis of NLP genes from Botrytis elliptica. Mol. Plant Pathol. 8:209-214.

Takami, H., Nakasone, K., Takaki, Y., Maeno, G., Sasaki, R., Masui, N., Fuji, F., Hirama, C., Nakamura, Y., Ogasawara, N., Kuhara S., and Horikoshi, K. 2000. Complete genome sequence of the alkaliphilic bacterium Bacillus halodurans and genomic sequence comparison with Bacillus subtilis. Nucleic Acids Res. 28:4317-4331.

Tiburcio, R. A., Costa, G. G., Carazzolle, M. F., Mondego, J. M., Schuster, S. C., Carlson, J. E., Guiltinan, M. J., Bailey, B. A., Mieczkowski, P., Meinhardt, L. W., and Pereira, G. A. G. 2010. Genes acquired by horizontal transfer are potentially involved in the evolution of phytopathogenicity in Moniliophthora perniciosa and Moniliophthora roreri, two of the major pathogens of cacao. J. Mol. Evol. 70:85-97.

Upadhyay, S. K., Gautam, P., Pandit, H., Singh, Y., Basir, S. F., and Madan, T. 2012. Identification of fibrinogen-binding proteins of Aspergillus fumigatus using proteomic approach. Mycopathologia 173:73-82.

Vega, F. E., Goettel, M. S., Blackwell, M., Chandler, D., Jackson, M. A., Keller, S., Koike, M., Maniania, N. K., Monzón, A., Ownley, B. H., Pell, J. K., Rangel, D. E. E., and Roy, H. E. 2009. Fungal entomopathogens: New insights on their ecology. Fungal Ecol. 2:149-59.

Veit, S., Worle, J. M., Nürnberger, T., Koch, W., and Seitz, H. U. 2001. A novel protein elicitor (PaNie) from Pythium aphanidermatum induces multiple defense responses in carrot, Arabidopsis, and tobacco. Plant Physiol. 127:832-841.

Wilson, I. B., Gavel, Y., and von Heijne, G. 1991. Amino acid distributions around O-linked glycosylation sites. Biochem. J. 275(Pt 2):529-34.

Wong, W., Maurer-Stroh, S., and Eisenhaber, F. 2010. More than 1,001 problems with protein domain databases: Transmembrane regions, signal peptides and the issue of sequence homology. PLoS Computational Biology 6:e1000867.

Yang, J. C., Madupu, R., Durkin, A. S., Ekborg, N. A., Pedamallu, C. S., Hostetler, J. B., Radune, D., Toms, B. S., Henrissat, B., Coutinho, P. M., Schwarz, S., Field, L., Trindade-Silva, A. E., Soares, C. A. G., Elshahawi, S., Hanora, A., Schmidt, E. W., Haygood, M. G., Posfai, J., Benner, J., Madinger, C., Nove, J., Anton, B., Chaudhary, K., Foster, J., Holman, A., Kumar, S., A., Lessard, P., Luyten, Y. A., Slatko, B., Wood, N., Wu, B., Teplitski, M., Mougous, J. D., Ward, N., Eisen, J. A., Badger, J. H., and Distel, D. L. 2009. The complete genome of Teredinibacter turnerae T7901: An intracellular endosymbiont of marine wood-boring bivalves (shipworms). PloS One 4:e6085. Published online.

Zaparoli, G., de Barsottini, M. R., de Oliveira, J. F., Dyszy, F., Teixeira, P. J., Barau, J. G., Garcia, O., Costa-Filho, A. J., Ambrosio, A. L. B., Pereira, G. A. G., and Dias, S. M. G. 2011. The crystal structure of necrosis- and ethylene-inducing protein 2 from the causal agent of cacao's witches' broom disease reveals key elements for its activity. Biochemistry 50:9901-9910.

Zhou, B.-J., Jia, P.-S., Gao, F., and Guo, H.-S 2012. Molecular characterization and functional analysis of a necrosis- and ethylene-inducing, protein-encoding gene family from Verticillium dahliae. Mol. PlantMicrobe Interact. 25:964-975.

\section{AUTHOR-RECOMMENDED INTERNET RESOURCES}

ClustalW software: www.ebi.ac.uk/Tools/msa/clustalw2

Consurf server: consurf.tau.ac.il

Endophyte Epichloë festucae genome project database: www.endophyte.uky.edu/ef

FigTree v1.4.0 server: tree.bio.ed.ac.uk/software/figtree FungiDB: fungidb.org

I-Tasser server: zhanglab.ccmb.med.umich.edu/I-TASSER

Joint Genome Institute database: genome.jgi-psf.org

National Center for Biotechnology Information BLAST database: blast.ncbi.nlm.nih.gov/Blast.cgi

SignalP4.1 server: www.cbs.dtu.dk/services/SignalP

Weblogo software: weblogo.berkeley.edu 